\title{
RECENT TRENDS IN NON-FARADAIC SUPERCAPACITOR ELECTRODE MATERIALS
}

\author{
Onyeka Stanislaus Okwundu *,1, 2, Cyril Oluchukwu Ugwuoke ${ }^{3}$, \\ Augustine Chukwujekwu Okaro ${ }^{3}$ \\ ${ }^{I}$ Department of Chemical and Petrochemical Engineering, Egypt-Japan University of \\ Science and Technology, Egypt \\ ${ }^{2}$ Department of Chemical Engineering, University of Benin, Nigeria \\ ${ }^{3}$ Department of Physics, University of Benin, Nigeria
}

Received 29.05.2019

Accepted 20.06.2019

\begin{abstract}
Global demand for energy is on a progressive increase and there is a need for environmentally friendly technologies to meet this demand. Electrochemical energy systems are hinged on clean and sustainable technologies. The latest trend in electrochemical energy systems is the supercapacitors (SCs). SCs are famous for their attractive properties: power density, charging time, life cycle, operational safety, and simplicity. However, their energy density is generally low and to a great extent, this parameter is invariably dependent on the nature of electrode material used. While high energy density is being sought for in SCs, it is necessary to keep abreast of recent electrode materials and their practical performances. This paper gives a concise description of capacitors with a focus on the non-Faradaic SCs. It also compendiously presents an overview of carbon electrode materials with their practical performances (specific surface area, specific capacitance, energy and power densities) for non-Faradaic SCs, with reference to more than 100 reputable works. Development and investigation of highly active carbon materials with optimized electrolytic compatibilities and manipulative morphologies and pore structures were recommended.
\end{abstract}

Keywords: ultracapacitors; EDLC; carbon electrode materials; energy storage; Helmholtz double layer.

\footnotetext{
* Corresponding author: Onyeka Stanislaus Okwundu, onyeka.okwundu@eng.uniben.edu; okwundu.onyeka@ejust.edu.eg
} 


\section{Contents}

1 Introduction 106

2. Advancement from Capacitors to SCs 107

2.1 Ordinary Capacitors 107

2.2 Concept of Helmholtz or Electric Double Layer (EDL) 109

2.3 Ultracapacitors or Supercapacitors (SCs) 110

3. Electrode Materials for Non-Faradaic SCs 112

4. Conclusion and Recommendations 132

Acknowledgement 132

References 132

\section{Introduction}

Progress in global industrialization alongside world population growth suggests that there would never be a slackening in global energy demand. Although several sources of energy are in place today, inadequate energy storage techniques and continuous depletion of non-renewable and non-sustainable fossil reserves coupled with the accompanying environmental pollution, call for sustainable and clean energy sources with efficient technologies for conversion and storage. Electrochemical energy conversion and storage systems which include secondary cells, supercapacitors (SCs) and fuel cells have proven to be one of the most effective, sustainable and eco-friendly panacea in this regard [1-4].

Electrochemical capacitors, also known as supercapacitors or ultracapacitors, are an advanced form of electrolytic capacitors. They possess some desirably salient features like high power density, long charge/discharge cycle, short charging time, a wide range of operating temperature, operational safety, and simplicity $[2,5]$. Considering the power and energy densities, they span from dielectric capacitors to secondary cells [6]. SCs have significantly caught the attention of researchers as evinced by numerous works published on this subject. Fig 1 presents the statistics of publications on SCs available from Elsevier's database (Scopus indexed publications) as at $23^{\text {rd }}$ May 2019. The publications include journal articles $(19,004)$, conference articles $(3,920)$, articles in press (accepted manuscripts, 302), book chapters (162), dissertations (147), books (15), errata (3) and a standard. It is evident from the bar chart that research interest in this area is increasing steadily. Meanwhile, an 'all-time' search on Google Scholar reveals approximately 221,000 and 196,000 publications, with and without patents, respectively. Nonetheless, SCs are quite expensive with relatively low energy density [5, 7]. 


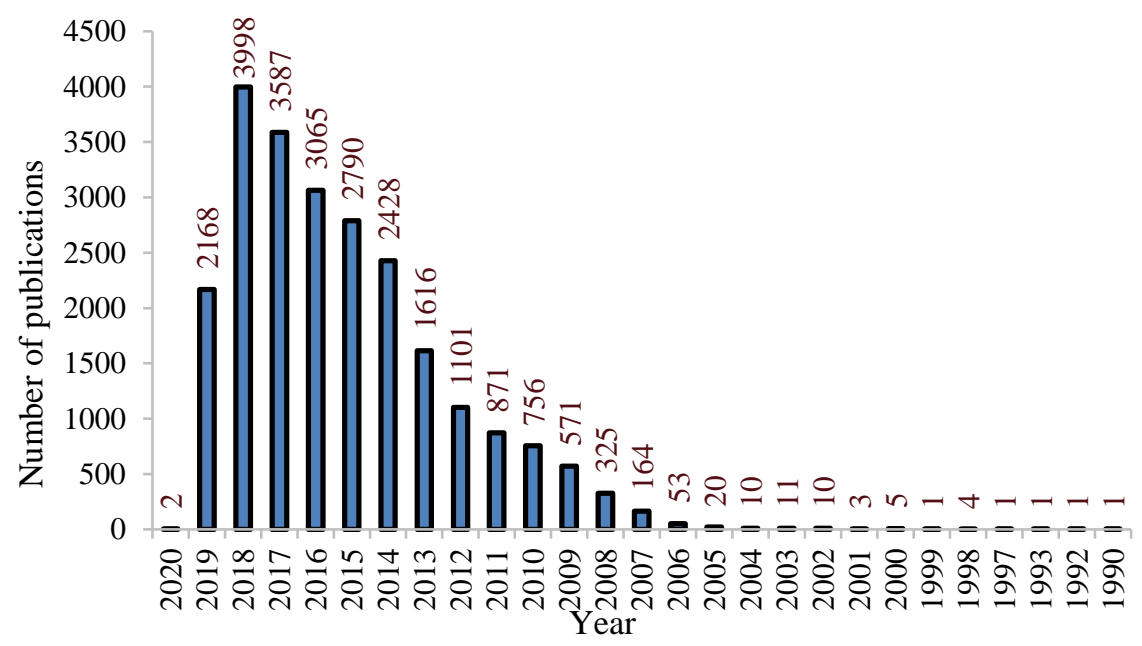

Fig. 1. Statistics of Scopus indexed publications on SCs as at $23^{\text {rd }}$ May 2019 (provided by Engineering Village (02019 Reed Elsevier).

Consequently, most studies have been geared towards improving the energy density of SCs. The expression for energy density (E) is as shown in Eq. 1, where: $\mathrm{C}$ is the capacitance of the SC and V is the potential drop across the double layers [2, 7]. It follows, therefore, that any effective attempt to increase the double layer capacitance or voltage would improve the energy density. Wu and Cao [7] utilized a schematic illustration to show that capacitance is affected by surface area, pore size, electrical conductivity, and available functional groups. Based on theoretical models, Yu et al. [2] noted that double layer capacitance of an SC is dependent on the quantity of charge, temperature, the concentration of electrolyte and solvent used; however, they went further to show that the nature of electrolyte and electrode used are of ultimate relevance.

$$
E=\frac{1}{2} C V^{2}
$$

Since the choice of electrode and electrolyte are of paramount importance for the performance of SCs, this paper reviews recent researches that employed carbon electrodes for SCs. In other words, only the pure or true electrostatic supercapacitors - those that do not exhibit Faradaic reactions - are covered here. For SC electrolytes, refer to the work of Zhong et al. [8].

\section{Advancement from Capacitors to SCs}

\section{Ordinary Capacitors}

A capacitor is a passive electric charge storage device comprising of two close conductors (electrodes) which are electrically insulated from each other. The insulating dielectric is usually sandwiched between the electrodes. Although the knowledge of static electricity has been since 600 BC; courtesy of Thales of Miletus [9], it was not until 1745 that the German bishop and scientist, Ewald Georg von Kleist invented the first capacitor (then known as condenser) after an unusual shock in an attempt to convey and store 
charges in a handheld glass jar filled with water [10]. After some months, Pieter van Musschenbroek, a Dutch physicist at the University of Leiden, coincidentally and independently, after a similar experience that made him write to French Mr. Reaumur, "I would not take a second shock for the kingdom of France", invented a similar capacitor which is now known as the Leyden Jar $[11,12]$. Thereafter, Benjamin Franklin simplified the design using flat glass piece as dielectric [12]. Fig 2 shows these earlier designs of the capacitor. Today, there are varieties of capacitor designs with variations in electrode geometry (parallel plates, co-axial cylinders, concentric spheres, and isolated sphere) and dielectric material (vacuum, non-ionized gases, insulating solids and liquids). Actually, the dielectric is the active charge storage component of a capacitor, as inferred by Franklin [13].
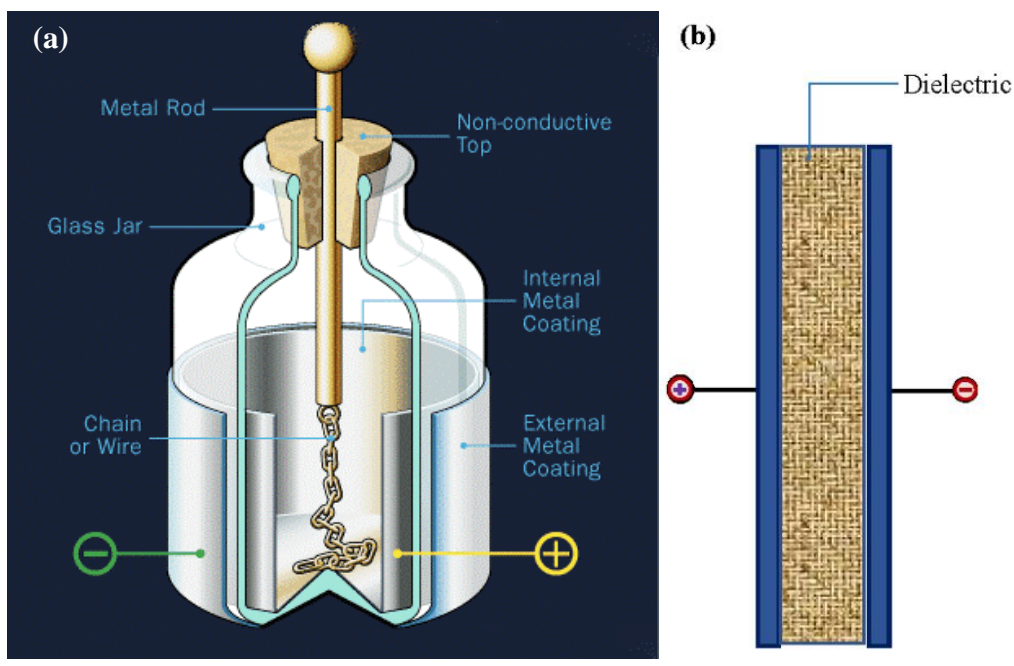

Fig. 2. Earlier designs of the capacitor: (a)- the Leyden jar [12] and (b)- parallel plate capacitor.

A measure of the amount of charge needed to induce a potential of 1 Volt across the electrodes of a capacitor is called the capacitance of the capacitor. For linear capacitors, this property is independent of the quantity of charge nor the potential difference, but upon the geometry of the device and the nature of dielectric used (permittivity, $\varepsilon$ ); larger electrode surface area (A) and shorter separation between charged layers or inter-electrode spacing (d) improve capacitance (C) as shown in Eq 2. Michael Faraday, an English chemist, demonstrated the first practical capacitor and how to use it for electric charge storage in his experiments. Faraday introduced a means of measuring the quantity of charge that can be held in a capacitor and this was measured in Farads, which is equivalent to Coulombs per Volt [12]. When the terminals of a capacitor are connected to a direct current source (charging mode), the source provides an electromotive force (emf) that drives electrons from the positive capacitor terminal to the positive source terminal, while simultaneously feeding electrons to the negative capacitor electrode at the same rate. Charging continues until the potential between the capacitor electrodes equates that of the emf source; then, the capacitor is fully charged concerning the driving potential [2]. During the discharge process, electrons flow in a reverse manner 
through a connected load; of course, the flow of electrons implies a flow of current but in reverse direction.

$$
C=\frac{\varepsilon A}{d}
$$

In 1896, Karol Franciszek (Charles) Pollak, a manufacturer of secondary cells filed a German patent (GB189601069) for an electric liquid capacitor with aluminum electrodes, after he discovered that the oxide layer on an aluminum anode remained stable in a neutral or alkaline electrolytic system after the circuit was opened [14]. He described his invention as a "liquid condenser with aluminum electrodes, which are covered with a uniformly insulating layer generated by forming with a weak current, characterized by using alkaline or neutral electrolyte. Since the insulating layer is very thin, the condenser has a very high capacitance and could be used as a polarized capacitor in a DC circuit." [15]. Therefore, electrolytic capacitors have thin insulating oxide films as their dielectric and they offer greater capacitance than the earlier types.

Capacitors are the most used electronic component after resistors. They are relevant in several applications, for instance in digital (electronic) circuits for information back-up, so that information stored in computer memories is not lost upon electric power failure; the stored energy in such capacitors maintains the information during temporary loss of power. Capacitors play an even more important role as filters for diversion of spurious electric signals, thereby preventing possible damage by electric surges to sensitive components of electric circuits, a typical example can be found in induction coils.

Concept of Helmholtz or Electric Double Layer (EDL)

When an insoluble charged or electrically active body is introduced into an electrolyte, there is to a certain degree, an alignment of the mobile ions (of the electrolyte) concerning the new charged surface. Electric Double Layer (EDL) is "a region existing at the boundary of two phases and assumed to consist of two oppositely charged layers (such as a layer of negative ions adsorbed on colloidal particles that attracts a layer of positive ions in the surrounding electrolytic solution)" [16]. Hermann von Helmholtz first realized this concept. He observed that a charged electrode immersed in electrolyte repels the co-ions while attracting the counterions to their surfaces; thereby forming 2 (double) layers of opposite polarity at the electrode-electrolyte interface [17]. This phenomenon was not recognized until 1879 [18]. The EDL is also referred to as the Helmholtz double layer. Helmholtz's concept has been developed successively by Gouy and Chapman; Stern; Grahame [2]; Bockris, Devanathan and Müller [19, 20]; and Conway [21].

Fig 3 is an illustration of double layer formation at the interface of negatively charged particle/rod and electrolyte. Static charges reside on the outer surface of materials, with higher concentrations at sharp edges. When introduced into the electrolyte, the material's charge distribution remains, while the mobile ions of the electrolyte get attracted (counterions) or repelled (co-ions) but the distribution of ions is somewhat diffuse - not as ideal as the material's surface charge distribution. A negatively charged insoluble material in an electrolyte attracts positively charged ions to its neighborhood to form an EDL. Electric double layers of negative (on the material surface) and diffuse positive charges can be seen in Fig 3; the layers are demarcated with a green 
dash line. The interlayer spacing is in the order of $1 \AA$ and more detailed description of this concept is given by Yu et al. [2], Park and Seo [22].

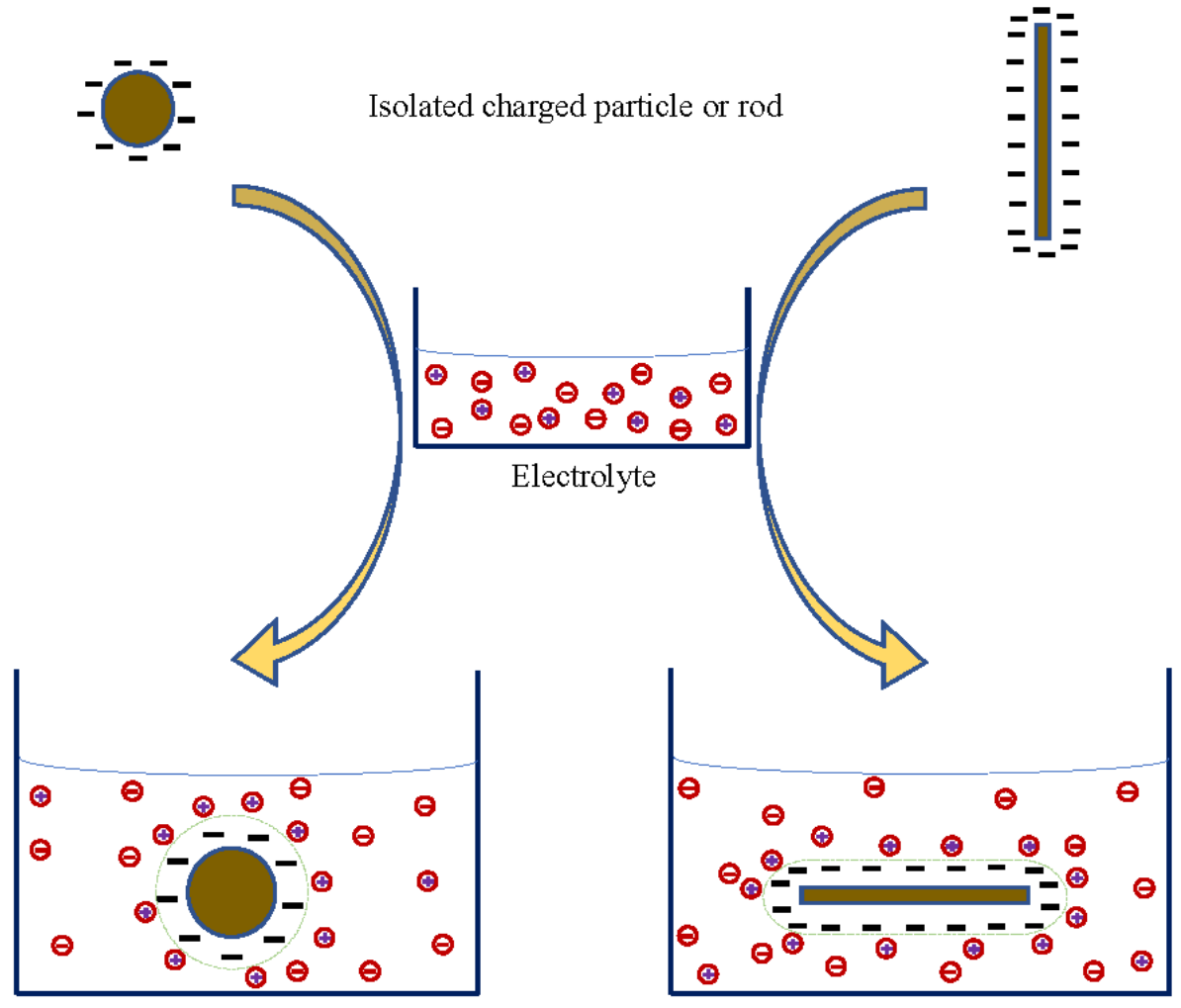

Fig. 3. Double layer formation at the interface of negatively charged particle/rod and electrolyte.

Ultracapacitors or Supercapacitors (SCs)

These are advanced or current generation capacitors with capacitance values much higher than a conventional capacitor. SCs can store 10 to 100 times Joules of energy per unit mass or volume when compared to the conventional capacitors [1]. While the ordinary (earlier generation) capacitors were developed for primary circuit applications, with storage of picofarad to microfarad charges in direct current circuits and filtration of frequencies in alternating current circuits, the current generation of capacitors, the SCs, are capable of serving more sophisticated purposes. Historical background of SCs can be found in the work of Samantara and Ratha [18].

A typical SC has two highly porous electrodes on a metallic collecting plate, electrolyte and an insulating membrane or separator between the electrodes that enables permeation of electrolyte ions but prevents contact/short-circuiting. The arrangement is similar to that of a secondary cell. Now, what makes SCs 'super' over ordinary capacitors? The SC electrodes are of extensive surface area in the order of $10^{3} \mathrm{~m}^{2} \mathrm{~g}^{-1}$ and the charge separation is typical of an EDL as earlier stated. Relating these unique features with Eq. 1 and 2, the prefix 'super' or 'ultra' would be found deserving of these advanced 
capacitors [23]. The fact that charge separation in SCs follows the EDL principle, has fetched SCs the name Electric Double Layer Capacitors (EDLCs). Fig 4 is a schematic diagram of a supercapacitor revealing the EDL at the non-intercalated electrolyteelectrode interface and the separation between charged layers (d) in reference to Eq. 2 . Since there is no permeation across the EDL interface (no charge intercalation), the tiny interface can be regarded as the dielectric for EDLCs but actually, they can be permeated as we would see in the case of pseudo-capacitors. The collectors are metallic plates onto which a porous substance (electrode material) such as activated charcoal or graphene nanoparticles is deposited. The large BET surface area of the electrode material offers SCs much more charge storage sites in an ideal situation where the electrolyte wets all the sites. However, in reality, all pores are never accessible [2, 8, 24].

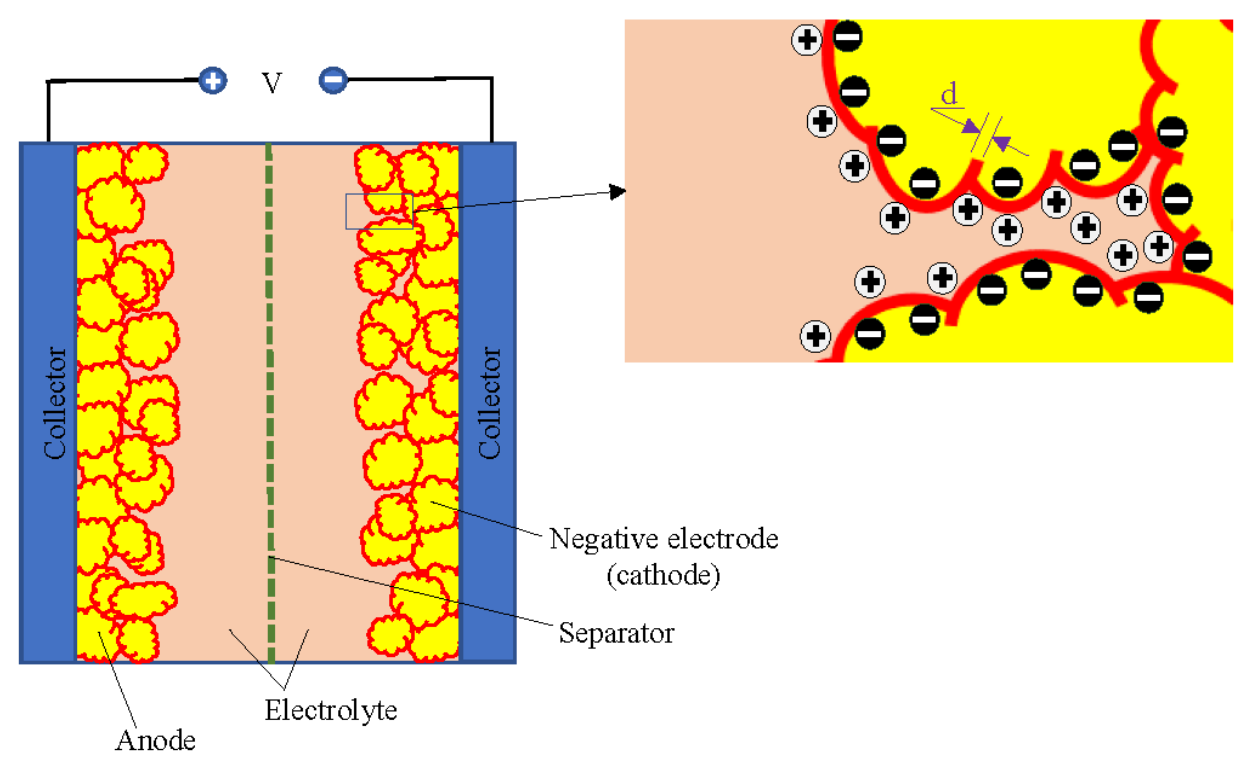

Fig. 4. Schematic diagram of a supercapacitor revealing the EDL formed at the electrolyte-electrode interface and the separation between charged layers (d) in reference to $E q .2$.

Furthermore, the mechanism of operation of SCs is best described alongside their classification. First, the 'true supercapacitors' store energy via physical adsorption of ions based on the EDL concept when an emf source is applied. Hence, charge storage is purely by electrostatic accumulation like in ordinary capacitors; non-Faradaic mechanism - no occurrence of a redox reaction. Consequently, this class of SCs are called non-Faradaic capacitors, electrostatic supercapacitors or even supercapacitors/EDLCs (since they are the true ones). This class of capacitors employ carbon electrodes and are characterized by relatively rapid charging/discharging, longer life cycle, and greater coulombic efficiencies than others $[2,5,24-26]$. Their merits come from the fact that the electrostatic mechanism is very much reversible and instantaneous.

Secondly, we have pseudocapacitors. They are the Faradaic capacitors, redox capacitors or the true electrochemical supercapacitors. The mechanism of 
pseudocapacitive charge storage is quite different from that of EDLCs. Here electrochemically active electrode (usually composed of transition metal oxide) material is used to bring about redox or Faradaic reaction. When connected to a source of emf, there is adsorption of ions, followed by fast and reversible redox reactions between the electrode materials and electrolyte, on the electrode interface. This reaction involves the intercalation or passage of charges across the EDL, resulting in a flow of Faradaic current, like the charging/discharging of secondary cells. Electrode materials for this class of supercapacitors include transition metal oxides and conducting polymers (such as polyaniline and polypyrrole) $[2,4,21,27,28]$. With conductive polymer-based electrodes, there is reversible electrochemical doping/de-doping [27]. Pseudocapacitors offer larger capacitance values and energy density than EDLCs, but they lag in power density due to the relatively slower Faradaic processes involved - reactions have to occur with intercalation of ions. They also suffer lower life cycle due to wear and tear from repeated Faradaic processes.

Lastly, hybrid supercapacitors are those formed by combining certain aspects of EDLCs with those of pseudocapacitors. Jain et al. [29] report the onset of Faradaic reaction in carbon electrode SC system with the introduction of p-hydroxyaniline to $1 \mathrm{M}$ aqueous sulphuric-acid electrolyte. This could be a lead to designing hybrid SCs with carbon electrodes using mixed electrolytes. Zhong et al. [8] utilized a schematic diagram to show these classes of a capacitor and they noted lithium ion capacitor as a typical hybrid SC. A detailed comparison between the Faradaic and non-Faradaic supercapacitors, as well as their contrasting features with secondary cells are covered in a technical paper by Conway [21].

Although SCs boast of high energy storage capacity compared to ordinary capacitors, they still lag behind batteries in that regard. Also, they are usually more expensive per unit than batteries $[5,30]$. On a technical note, it is possible to replace a cell phone battery with an SC, and it will charge much faster, but it can only stay charged for a while. SCs are very good at acquiring or delivering a sudden surge of energy and this makes them right candidate for hybrid energy systems with primary energy sources like the internal combustion engines, secondary and fuel cells which are capable of producing energy continuously but at low power. For instance, in powering an electric car, an SC can provide the power for acceleration, while a battery provides over the range of uniform motion and recharges the SC in-between surges [30].

\section{Electrode Materials for Non-Faradaic SCs}

Carbon-based materials have some salient features such as high chemical stability, structural polymorphism, low cost, wide potential windows, relatively inert electrochemistry, rich surface chemistry or electro-catalytic activities for a variety of redox reactions [31-33]. For this versatile nature, varieties of carbonaceous materials have been developed and studied extensively for several electrochemical applications; more importantly, for energy generation/conversion and storage [31, 34, 35]. Use of graphene-based materials in electrochemistry has been reviewed by Chen et al. [36].

Electrode materials for SCs are desired to be highly porous with electrolyteaccessible pores, electrically conducting and cheap. More specifically, non-Faradaic SC electrodes are desired to be electrochemically inert and this makes carbon materials a perfect candidate. Techniques for synthesis of non-Faradaic SC electrode materials include carbonization of biomaterials, followed by surface enhancement via thermal 
or/and CA, chemical vapor deposition [35, 37], electrospinning [28], electrodeposition of electrode films via an electrolytic coating, direct coating, etc. [38]. In order to create abundant micro-, meso- or macro-pores within the carbon material, soft and/or hard templates are usually employed in the carbonization process, after which they are removed without further chemical/thermal activation (CA or TA). This is known as template carbonization method [39]. Methods of electrode performance assessment are described in the review work of Stoller and Ruoff [40].

Finally, we present some trending carbon-based materials that have been tried as electrodes in supercapacitors with their practical performances: specific surface area, capacitance, energy and power. In the consolidation of our earlier review [35], this work reveals one of the unlimited potentials of carbon. Review on this topic has been done before [3, 38, 41-43], but for the first time, we compose concisely, practical performance results of numerous carbon electrodes for non-Faradaic SCs. Table 1 shows the performance of carbon electrode materials in SCs. 


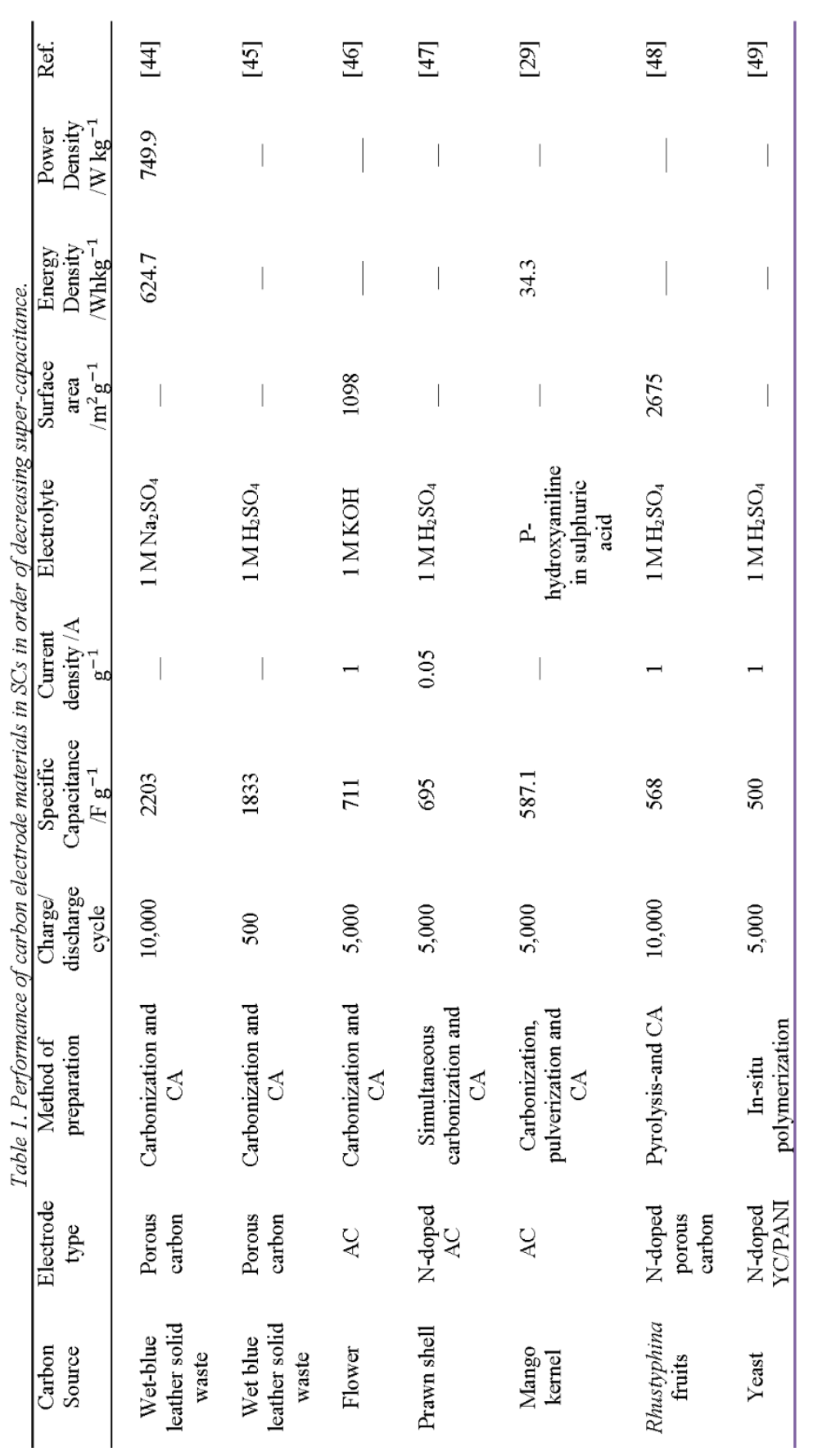




\begin{tabular}{|c|c|c|c|c|c|c|c|c|c|}
\hline$\stackrel{\bar{n}}{ }$ & 垔 & $\overline{\underline{n}}$ & $\bar{\Omega}$ & $\bar{n}$ & $\underset{\sim}{\stackrel{F}{n}}$ & $\sqrt{n}$ & 官 & $\sqrt{n}$ & $\stackrel{\infty}{\stackrel{\infty}{n}}$ \\
\hline 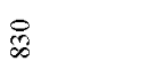 & & 1 & 1 & I & శે & 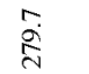 & 1 & 1 & 1 \\
\hline 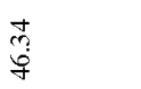 & & 1 & 1 & 1 & $\stackrel{\overbrace{}}{i}$ & $\begin{array}{l}\text { m } \\
\text { à }\end{array}$ & I & 1 & I \\
\hline & 롱 & 1 & ત્તે & 怘 & $\begin{array}{l}\stackrel{3}{0} \\
\stackrel{4}{n} \\
i\end{array}$ & $\frac{\bar{a}}{\overrightarrow{1}}$ & 1 & $\begin{array}{l}\infty \\
\stackrel{\infty}{\sim} \\
\stackrel{\sim}{*}\end{array}$ & 1 \\
\hline 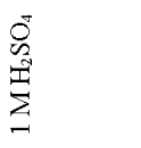 & 薄 & 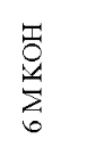 & 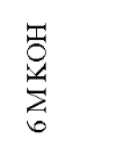 & $\underset{3}{3}$ & 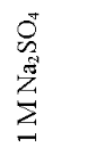 & $\begin{array}{l}\Xi \\
0 \\
\vdots \\
\vdots \\
\vdots\end{array}$ & 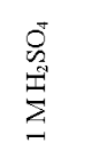 & $\sum_{0}^{5}$ & 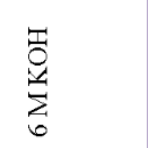 \\
\hline$N$ & - & $\overrightarrow{0}$ & 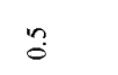 & $\stackrel{\circ}{-}$ & $\because$ & - & - & ?n & $\because$ \\
\hline 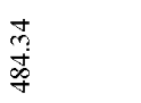 & $\underset{f}{\stackrel{t}{f}}$ & $\widehat{\nearrow}$ & $\stackrel{n}{\xi}$ & $\stackrel{m}{F}$ & \& & 夺 & $\tilde{\sigma}$ & 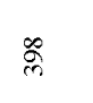 & $\overline{\mathrm{e}}$ \\
\hline & $\begin{array}{l}8 \\
\stackrel{0}{\circ}\end{array}$ & $\stackrel{8}{-}$ & . & 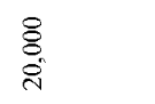 & 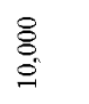 & $\underset{\infty}{\$}$ & $\begin{array}{l}8 \\
\stackrel{8}{0}\end{array}$ & $\begin{array}{l}8 \\
\stackrel{0}{\circ}\end{array}$ & $\stackrel{8}{8}$ \\
\hline 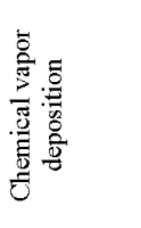 & 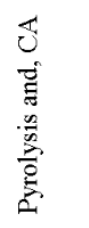 & 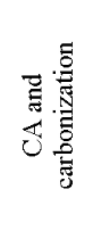 & 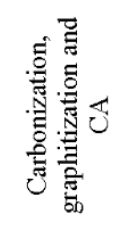 & 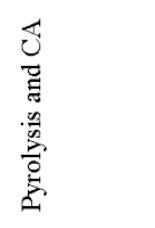 & 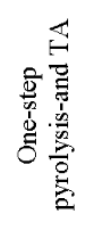 & 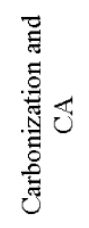 & 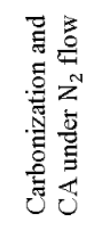 & 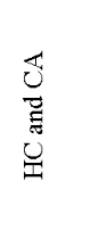 & 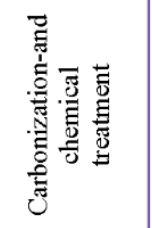 \\
\hline 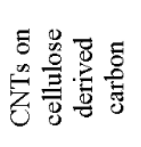 & 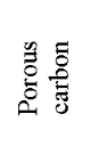 & $\underbrace{\infty}_{\dot{Z}}$ & 害 & 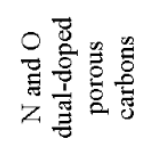 & 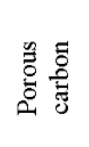 & ¿ & 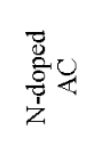 & 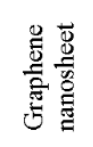 & 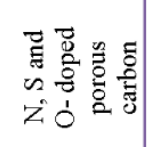 \\
\hline 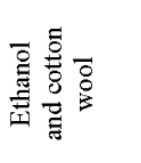 & 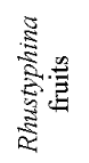 & 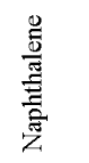 & 崖 & 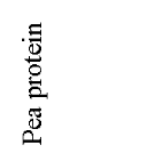 & 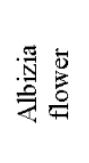 & 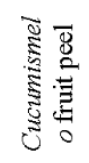 & $\frac{\frac{8}{0}}{0}$ & 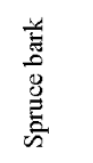 & 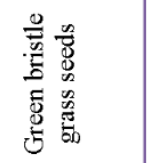 \\
\hline
\end{tabular}




\begin{tabular}{|c|c|c|c|c|c|c|c|c|}
\hline פू & $\bar{\sigma}$ & $\vec{\sigma}$ & $\overline{\widetilde{\sigma}}$ & 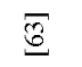 & 悉 & 厚 & 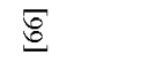 & $\sqrt{6}$ \\
\hline \pm & 1 & ث্ & & & I & $\bar{q}$ & I & I \\
\hline 2 & I & $\stackrel{\vec{\gamma}}{\vec{j}}$ & $\begin{array}{l}8 \\
\dot{b} \\
\dot{g}\end{array}$ & & I & $\stackrel{\sim}{\sigma}$ & $\underset{\mathbb{J}}{\stackrel{J}{J}}$ & $\approx$ \\
\hline$\stackrel{\infty}{\circ}$ & ఫ્. & | & $\underset{\exists}{\stackrel{J}{J}}$ & $\begin{array}{l}\stackrel{\ominus}{\dot{J}} \\
\stackrel{\vec{\Xi}}{\Xi}\end{array}$ & 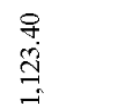 & $\stackrel{\infty}{\stackrel{\infty}{\sim}}$ & I & 1 \\
\hline 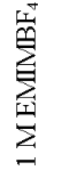 & I & 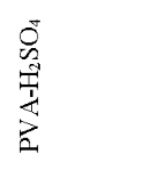 & $\begin{array}{l}0 \\
0 \\
\vdots \\
\vdots \\
\vdots \\
-1\end{array}$ & 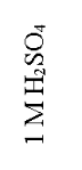 & $\underbrace{a}_{0}$ & 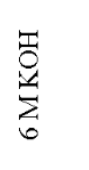 & 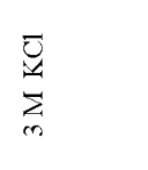 & 营 \\
\hline$\because$ & $\because$ & I & ฮี & $\cong$ & - & - & - & $\overline{0}$ \\
\hline \& & \& & \&్ల & 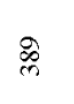 & 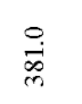 & in & 尺े & 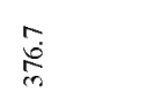 & 点 \\
\hline & $\stackrel{8}{8}$ & 1 & $\stackrel{8}{8}$ & $\underset{8}{8}$ & $\stackrel{8}{\stackrel{8}{\circ}}$ & 1 & $\stackrel{8}{8}$ & 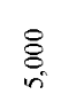 \\
\hline 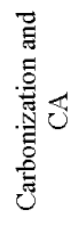 & 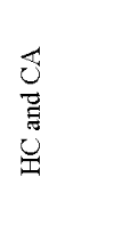 & 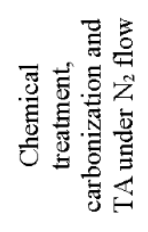 & 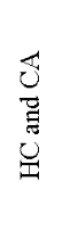 & 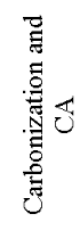 & 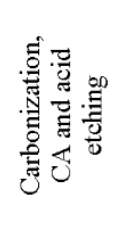 & 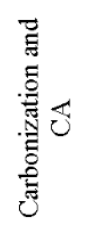 & 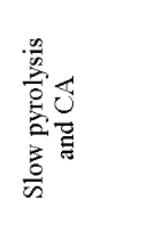 & 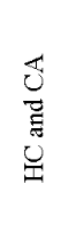 \\
\hline Z & 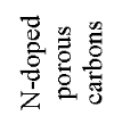 & $\begin{array}{l}\vec{y} \\
\grave{z}\end{array}$ & 音 & Y & 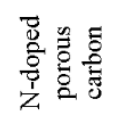 & 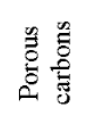 & 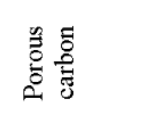 & 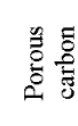 \\
\hline $\begin{array}{l}\text { 웡 } \\
\text { : }\end{array}$ & 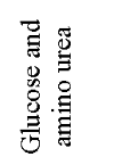 & 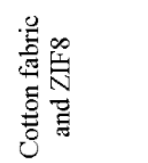 & 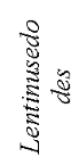 & 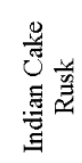 & 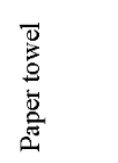 & 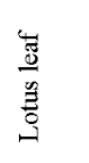 & 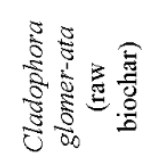 & 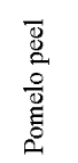 \\
\hline
\end{tabular}




\begin{tabular}{|c|c|c|c|c|c|c|c|c|c|}
\hline 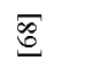 & 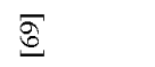 & $\bar{\Xi}$ & $\Xi$ & $\bar{\Xi}$ & $\stackrel{乛}{\subseteq}$ & 导 & $\underset{\Xi}{\Xi}$ & $\Sigma$ & 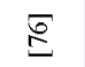 \\
\hline \&్ల & 1 & 옴 & $\underset{\infty}{\stackrel{\infty}{\infty}}$ & I & I & 1 & I & & $\stackrel{8}{\circ}$ \\
\hline 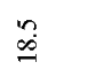 & ¿ి & $\hat{\infty}$ & $\stackrel{+}{\stackrel{d}{d}}$ & I & $\stackrel{a}{r}$ & I & 1 & & $\underset{\dot{m}}{\mathbb{d}}$ \\
\hline$\stackrel{0}{\circ}$ & I & $\underset{⿱ t}{t}$ & 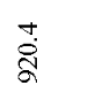 & $\stackrel{尺}{尺}$ & $\begin{array}{l}\infty \\
\stackrel{0}{0} \\
\stackrel{d}{0}\end{array}$ & I & I & & 1 \\
\hline 象 & 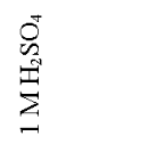 & $\underset{0}{3}$ & | & 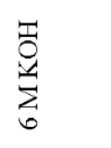 & 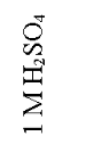 & 空 & 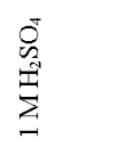 & 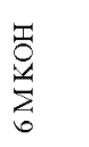 & 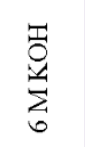 \\
\hline$n$ & I & 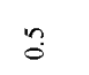 & - & $\dddot{n}$ & $\dddot{n}$ & $\stackrel{\leftrightarrow}{0}$ & $\stackrel{\dddot{a}}{\stackrel{0}{0}}$ & - & - \\
\hline$\stackrel{8}{\infty}$ & 总 & $\underset{ల}{b}$ & $\begin{array}{l}\text { ్․ } \\
\text { ర్లn }\end{array}$ & 品 & 总 & 点 & 点 & în & 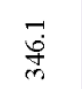 \\
\hline$\AA$ & $\stackrel{8}{8}$ & $\stackrel{8}{8}$ & | & $\stackrel{8}{8}$ & $\begin{array}{l}8 \\
\text { in }\end{array}$ & I & I & & \&̊․ \\
\hline 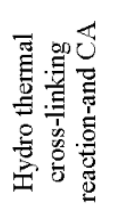 & 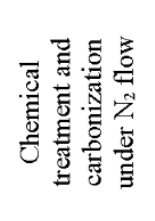 & 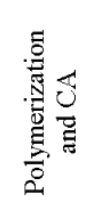 & 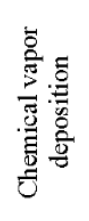 & 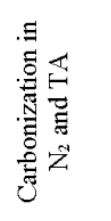 & 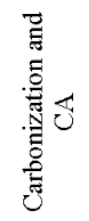 & 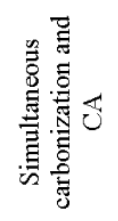 & 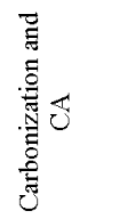 & 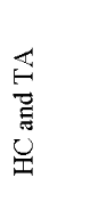 & 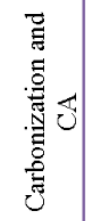 \\
\hline 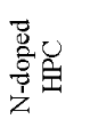 & 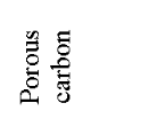 & $\begin{array}{l}\overrightarrow{D_{0}} \\
\text { 覫 }\end{array}$ & 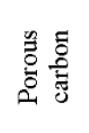 & 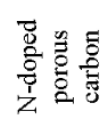 & 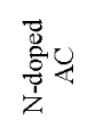 & 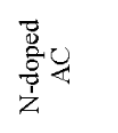 & 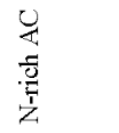 & 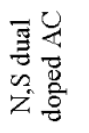 & 安 \\
\hline 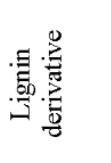 & 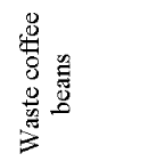 & 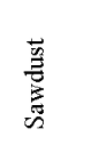 & 惫 & 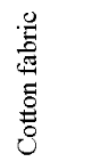 & 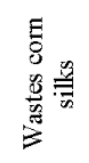 & 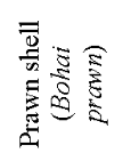 & 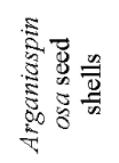 & 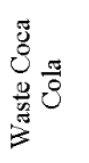 & 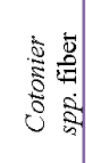 \\
\hline
\end{tabular}




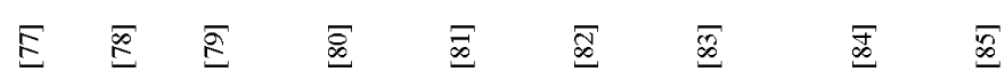

赵我

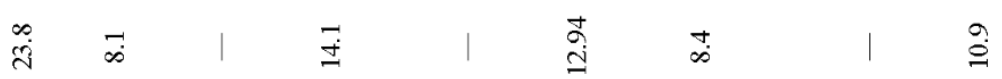

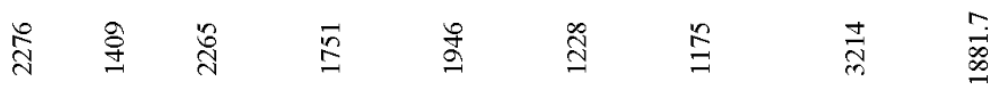

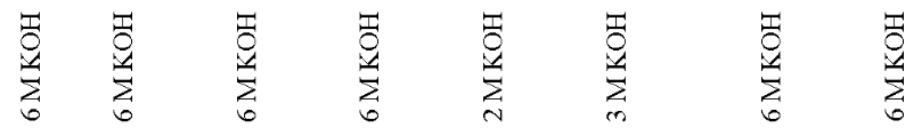

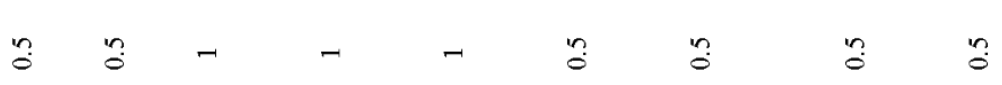

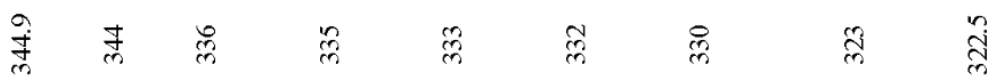

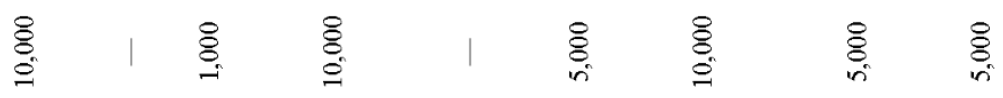

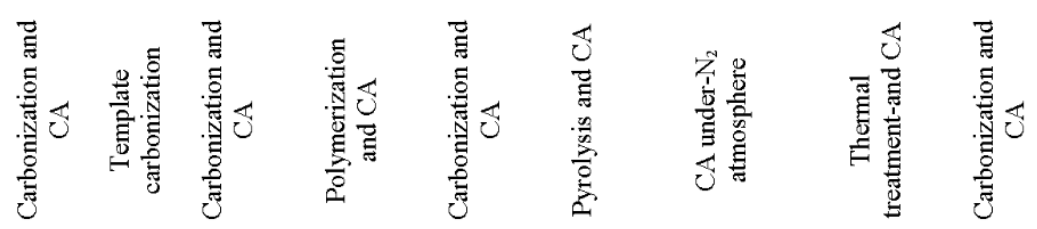

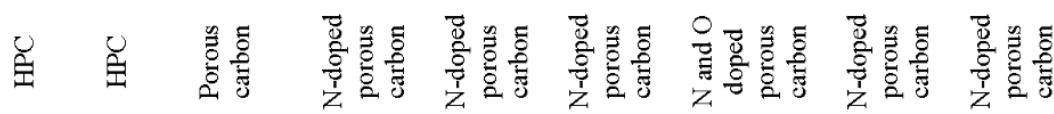

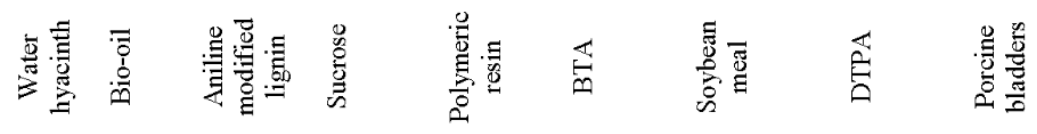




\begin{tabular}{|c|c|c|c|c|c|c|c|c|c|}
\hline$\underset{\mathscr{D}}{\mathscr{\infty}}$ & $\underset{\infty}{\infty}$ & $\stackrel{\mathscr{\infty}}{\stackrel{\mathscr{\infty}}{ }}$ & $\underset{\mathscr{D}}{\bar{\alpha}}$ & $\bar{\Xi}$ & $\underline{\bar{\sigma}}$ & $\bar{\Xi}$ & $\widetilde{\Omega}$ & হ్ & F \\
\hline $\mathfrak{g}$ & $\stackrel{\widetilde{\infty}}{\stackrel{\infty}{\prime}}$ & I & I & ฌั & I & I & $\hat{\mathrm{i}}$ & $\begin{array}{l}\mathbb{0} \\
\stackrel{0}{0}\end{array}$ & 1 \\
\hline$\stackrel{3}{\mathrm{~N}}$ & ฉิ & I & I & $\stackrel{\sim}{\sim}$ & 1 & 1 & $\stackrel{\circ}{=}$ & $\stackrel{\Re}{\stackrel{\varrho}{\varrho}}$ & 1 \\
\hline $\mid$ & సે & $\begin{array}{l}\stackrel{\circ}{0} \\
\stackrel{\Xi}{\Xi}\end{array}$ & $\begin{array}{l}\ddot{\infty} \\
\stackrel{\sim}{N}\end{array}$ & $\stackrel{\infty}{\infty}$ & 竒 & $\begin{array}{l}n \\
\stackrel{n}{\sigma} \\
g\end{array}$ & $\frac{8}{m}$ & 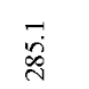 & 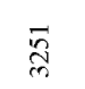 \\
\hline $\begin{array}{l}3 \\
z \\
z\end{array}$ & 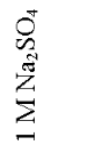 & $\begin{array}{l}3 \\
0 \\
0 \\
0 \\
0\end{array}$ & 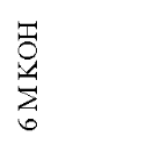 & 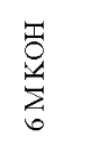 & 焉 & 䓂 & $\begin{array}{l}\underbrace{}_{0} \\
\vdots \\
0\end{array}$ & 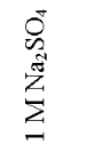 & 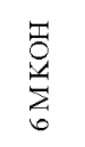 \\
\hline- & $n$ & ชี & $\stackrel{n}{0}$ & $\ddot{n}$ & $\stackrel{n}{0}$ & $\because$ & $\because$ & I & $\stackrel{8}{\circ}$ \\
\hline ¿ั. & స్ & $\stackrel{0}{m}$ & $\underset{m}{\Delta}$ & $\frac{m}{m}$ & ले & $\frac{7}{m}$ & $\stackrel{n}{\stackrel{n}{0}}$ & $\begin{array}{l}\text { 今े } \\
\text { d. } \\
\text { d. }\end{array}$ & 总 \\
\hline $\begin{array}{l}8 \\
\text { in } \\
\text { in }\end{array}$ & $\begin{array}{l}8 \\
8 \\
\text { :n }\end{array}$ & $\underset{8}{8}$ & $\stackrel{8}{8}$ & 1 & $\underset{8}{8}$ & $\stackrel{\S}{\AA}$ & & $\underset{8}{8}$ & $\stackrel{8}{8}$ \\
\hline 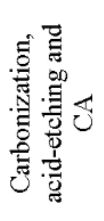 & 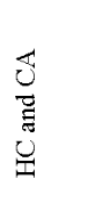 & 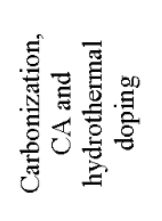 & 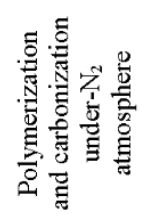 & 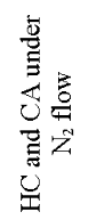 & 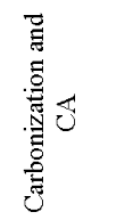 & 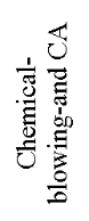 & 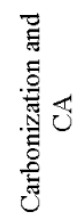 & 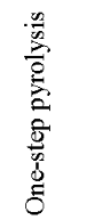 & 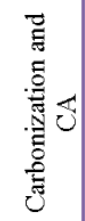 \\
\hline U & 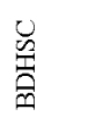 & 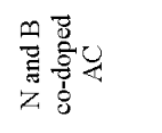 & 密 & 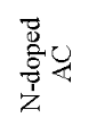 & 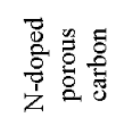 & 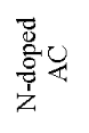 & 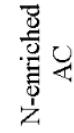 & 兰 & \\
\hline 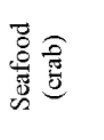 & 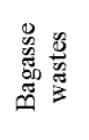 & 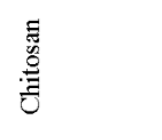 & 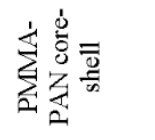 & 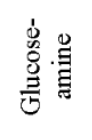 & 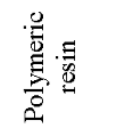 & 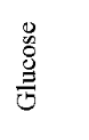 & 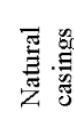 & 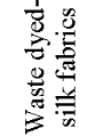 & 言豆 \\
\hline
\end{tabular}




\begin{tabular}{|c|c|c|c|c|c|c|c|}
\hline $\bar{a}$ & $\Phi$ & $\Xi$ & $\stackrel{\bar{g}}{a}$ & $\bar{\alpha}$ & $\stackrel{\Xi}{\varrho}$ & $\bar{\Xi}$ & 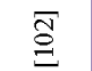 \\
\hline$\stackrel{n}{8}$ & 1 & I & I & $\stackrel{\otimes}{\oplus}$ & I & 1 & $\underset{\substack{i \\
\infty}}{i d}$ \\
\hline$\stackrel{\sim}{2}$ & 1 & $\overrightarrow{6}$ & $\hat{i}$ & $\stackrel{\stackrel{\vec{N}}{ }}{ }$ & I & 1 & $\stackrel{\circ}{\dot{q}}$ \\
\hline$\stackrel{\infty}{\stackrel{\Xi}{\Xi}}$ & $\hat{\alpha}$ & 社 & I & । & 窎命 & શે & | \\
\hline 㫄 & $\begin{array}{l}\text { 竞 } \\
\stackrel{3}{3} \\
6\end{array}$ & 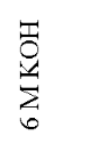 & 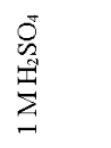 & $\underbrace{\frac{\pi}{0}}_{0}$ & $\sum_{0}^{3}$ & 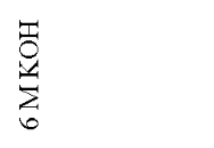 & $\begin{array}{l}\text { 薄 } \\
\stackrel{3}{\mid}\end{array}$ \\
\hline$\stackrel{\sim}{\circ}$ & | & 7 & - & $\because$ & $\stackrel{n}{0}$ & $\because$ & - \\
\hline 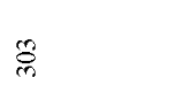 & ర్ల & हे & : & 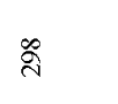 & ถิ & 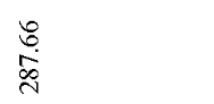 & $\overrightarrow{\overrightarrow{0}}$ \\
\hline$\stackrel{8}{\circ}$ & $\stackrel{8}{\circ}$ & 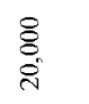 & $\underset{8}{8}$ & $\begin{array}{l}8 \\
\stackrel{0}{\circ}\end{array}$ & ڤ & \& & $\begin{array}{l}8 \\
\stackrel{8}{0}\end{array}$ \\
\hline 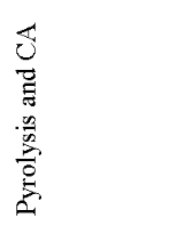 & 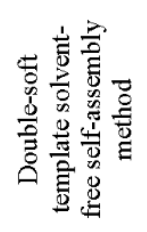 & 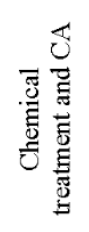 & 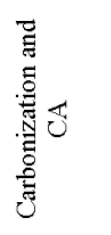 & 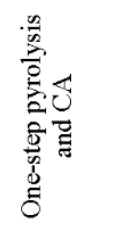 & 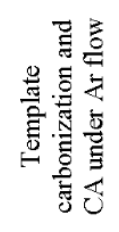 & 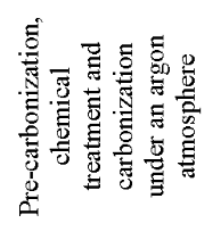 & 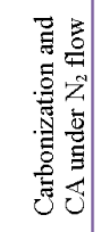 \\
\hline 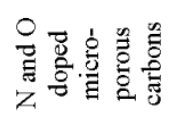 & 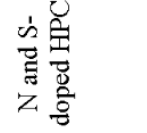 & 总 & 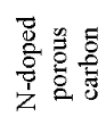 & 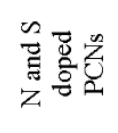 & $\sum_{0}^{n}$ & 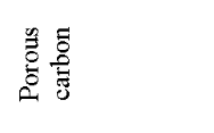 & 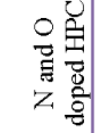 \\
\hline 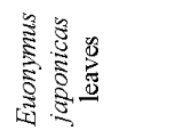 & 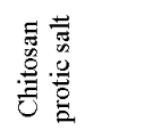 & 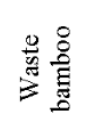 & 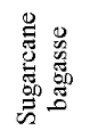 & 言具 & 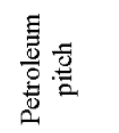 & 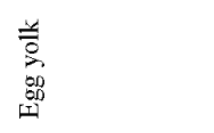 & $\begin{array}{l}\text { 素 } \\
\frac{0}{0.0} \\
2 \\
2\end{array}$ \\
\hline
\end{tabular}




\begin{tabular}{|c|c|c|c|c|c|c|c|c|}
\hline$\stackrel{\bar{\rho}}{\stackrel{\rho}{\varrho}}$ & $\stackrel{\vec{O}}{\Theta}$ & $\stackrel{\bar{\varrho}}{\varrho}$ & 足 & $\underset{\varrho}{E}$ & $\stackrel{\infty}{\varrho}$ & $\stackrel{\bar{g}}{g}$ & $\underset{\Omega}{\sigma}$ & $\stackrel{\Xi}{\Xi}$ \\
\hline | & | & 1 & I & \& & | & | & 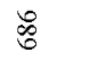 & | \\
\hline | & $\stackrel{m}{m}$ & 1 & I & 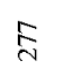 & ì & 1 & 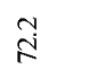 & $\begin{array}{l}\overrightarrow{3} \\
8\end{array}$ \\
\hline$\underset{n}{\stackrel{n}{c}}$ & 1 & $\stackrel{\check{a}}{\mathrm{a}}$ & ڤે & $\stackrel{\infty}{\infty}$ & $\stackrel{\mathscr{2}}{\Omega}$ & I & $\underset{0}{\ddot{8}}$ & I \\
\hline 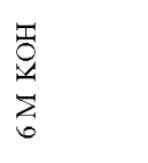 & 营 & 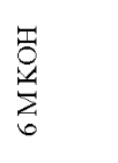 & 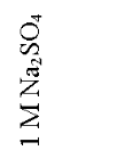 & 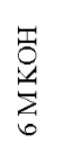 & | & 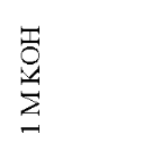 & 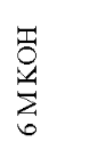 & 竞 \\
\hline$\stackrel{\text { ฮุ }}{0}$ & $\stackrel{n}{0}$ & - & $\stackrel{n}{0}$ & $\stackrel{n}{o}$ & $\stackrel{n}{\sim}$ & $\stackrel{\leftrightarrow}{-}$ & - & | \\
\hline$\hat{\stackrel{D}{0}}$ & $\begin{array}{l}\stackrel{0}{\circ} \\
\stackrel{\infty}{\sim}\end{array}$ & $\stackrel{\mathscr{8}}{\sim}$ & $\ddot{\otimes}$ & ㅊ & $\begin{array}{l}\vec{m} \\
\stackrel{\sim}{\sim}\end{array}$ & $\begin{array}{l}\text { ్․ } \\
\text { ה్ }\end{array}$ & 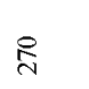 & $\stackrel{\infty}{\sim}$ \\
\hline 1 & $\begin{array}{l}\stackrel{8}{\circ} \\
\stackrel{-}{-1}\end{array}$ & ¿̊. & $\begin{array}{l}\text { \& } \\
\text { ¿̂. }\end{array}$ & 1 & 离 & 兽 & $\stackrel{8}{8}$ & $\begin{array}{l}8 \\
8 \\
n \\
n\end{array}$ \\
\hline 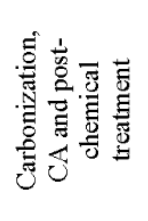 & 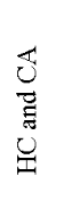 & 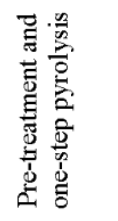 & 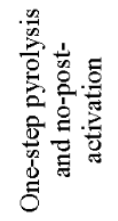 & 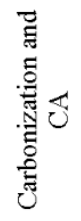 & 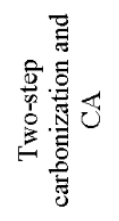 & 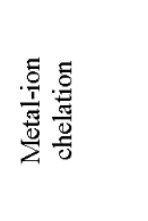 & 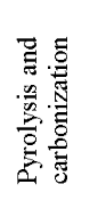 & 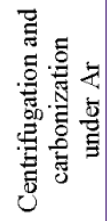 \\
\hline 著 & 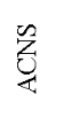 & 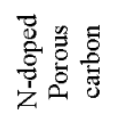 & 岕 & U & 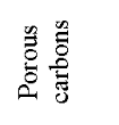 & 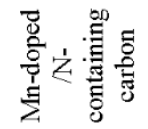 & 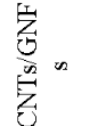 & 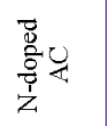 \\
\hline 惡 & 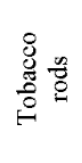 & 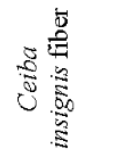 & 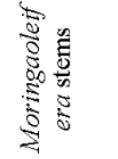 & 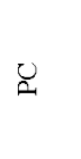 & 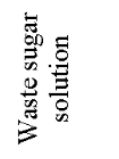 & 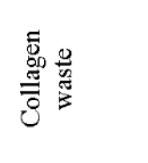 & 蛋 & 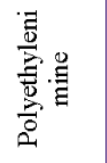 \\
\hline
\end{tabular}




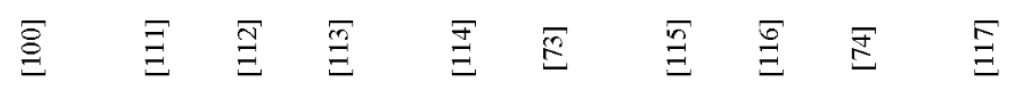

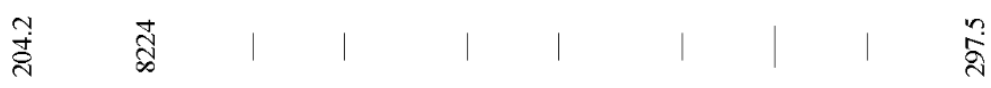

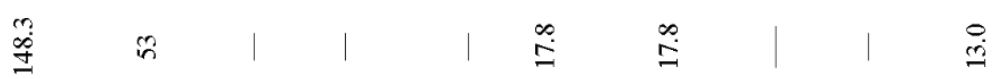

$$
\begin{aligned}
& \text { 窇总离 总 } \\
& \text { 息 }
\end{aligned}
$$

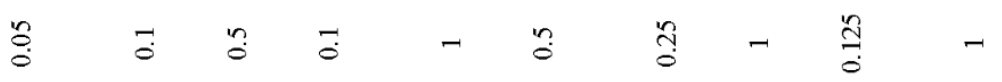

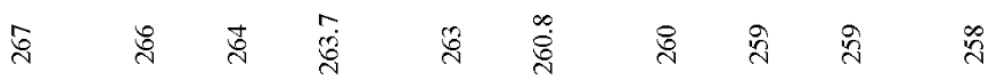

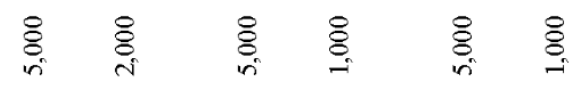

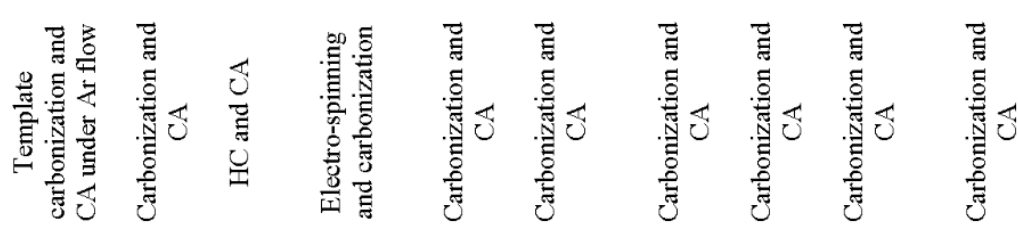

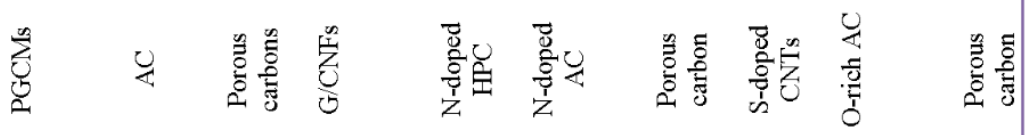

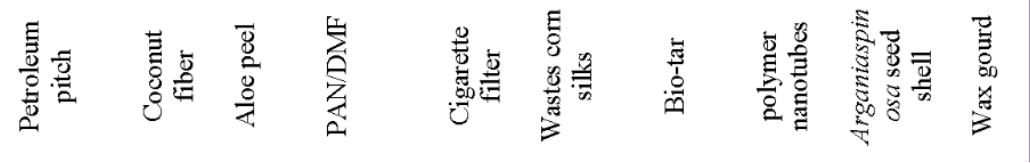




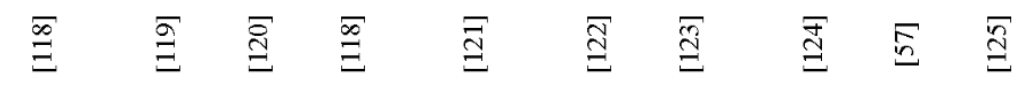

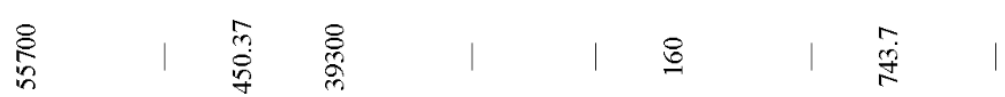

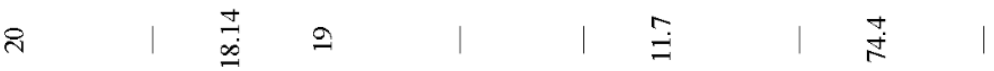

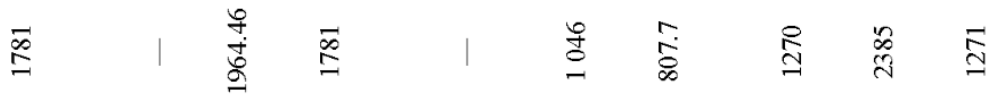

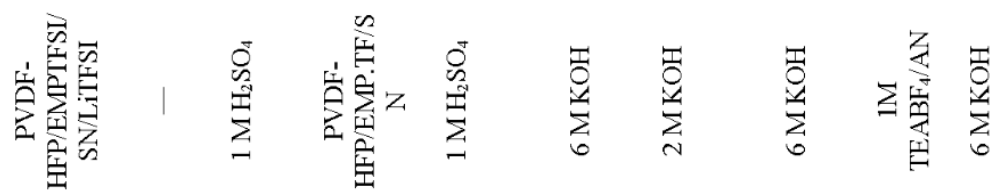

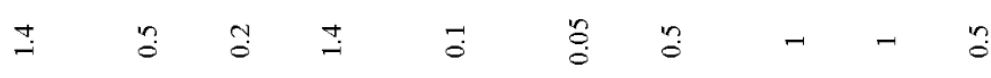

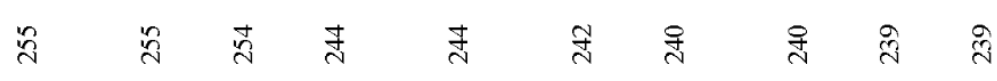

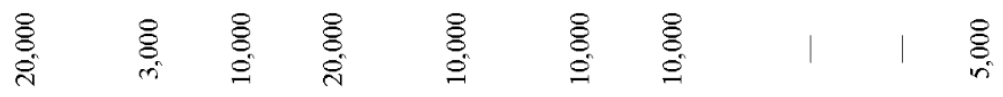

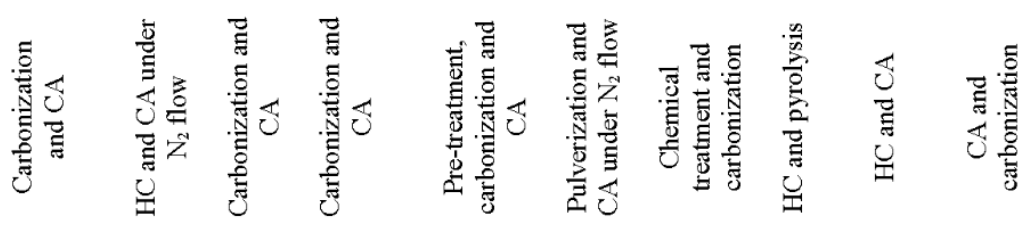

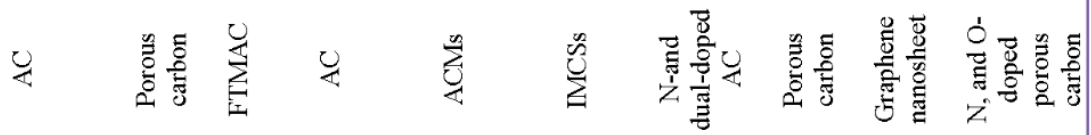

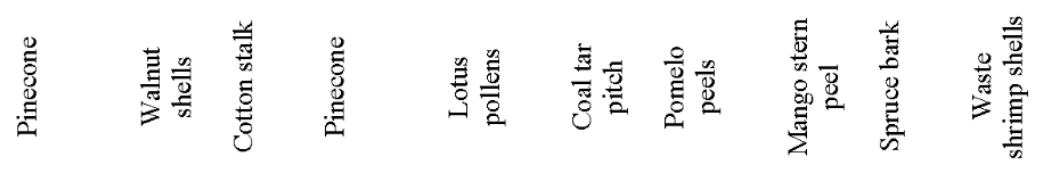




\begin{tabular}{|c|c|c|c|c|c|c|c|c|}
\hline 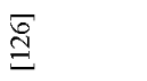 & $\underset{\Xi}{\Xi}$ & $\stackrel{\overline{\mathscr{D}}}{\Xi}$ & $\overline{\mathrm{d}}$ & 窎 & $\bar{\Xi}$ & $\overline{8}$ & $\begin{array}{l}\widetilde{\Xi} \\
\stackrel{\Xi}{ }\end{array}$ & $\stackrel{\bar{m}}{\stackrel{3}{\Xi}}$ \\
\hline | & I & 1 & 1 & I & & 尌 & | & $\stackrel{\circ}{\vec{i}}$ \\
\hline | & I & I & 1 & I & $\stackrel{ \pm}{\Xi}$ & $\vec{r}$ & I & స̊ \\
\hline | & $\frac{8}{\sqrt{2}}$ & 1 & 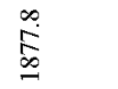 & $\bar{\Xi}$ & స్లి & 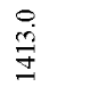 & $\underset{\text { dे }}{\text { d }}$ & $\underset{d}{\stackrel{+}{d}}$ \\
\hline 無 & 蒙 & 㫄 & $\begin{array}{l}\underbrace{}_{0} \\
\vdots \\
\vdots \\
6\end{array}$ & 焉 & $\begin{array}{l}3 \\
0 \\
0 \\
0 \\
0\end{array}$ & 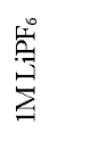 & 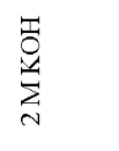 & 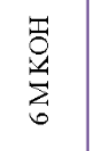 \\
\hline$\because$ & $\overrightarrow{0}$ & $\overrightarrow{0}$ & - & $\stackrel{\circ}{\circ}$ & $\infty$ & $\stackrel{m}{m}$ & - & | \\
\hline 号 & సે & జ̊ & జ̊ & సี & $\stackrel{\infty}{\grave{N}}$ & $\underset{\stackrel{\sim}{\sim}}{\stackrel{\circ}{\sim}}$ & $\stackrel{\sim}{\sim}$ & $\stackrel{\stackrel{\sim}{~}}{\stackrel{\sim}{\sim}}$ \\
\hline $\begin{array}{l}8 \\
\text { ¿n } \\
\text { in }\end{array}$ & $\begin{array}{l}\text { ¿े } \\
\text { ¿. }\end{array}$ & $\stackrel{8}{\circ}$ & & $\stackrel{8}{\circ}$ & & $\stackrel{8}{8}$ & $\stackrel{8}{\circ}$ & $\begin{array}{l}8 \\
\varrho\end{array}$ \\
\hline 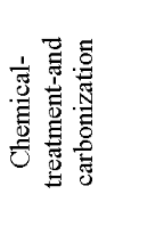 & 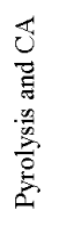 & 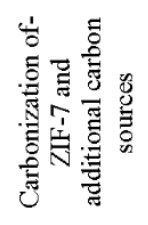 & 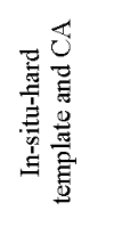 & 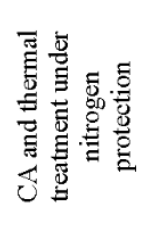 & 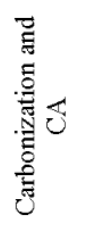 & 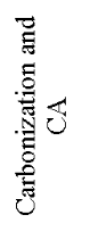 & 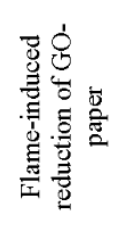 & 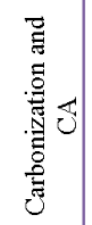 \\
\hline 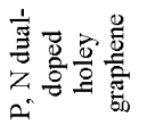 & 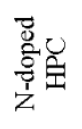 & 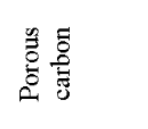 & 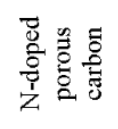 & 舫 & 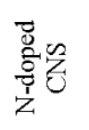 & U & $\stackrel{8}{1}$ & 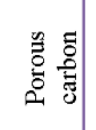 \\
\hline $\begin{array}{l}0 \\
\text { 总 } \\
\frac{0}{5}\end{array}$ & 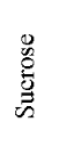 & 䇆 & 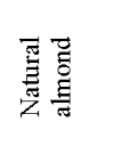 & $\begin{array}{l}\frac{0}{0} \\
\stackrel{3}{3} \\
\simeq 4\end{array}$ & 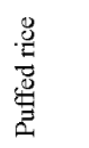 & 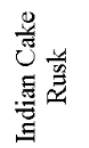 & $\begin{array}{l}\text { 总 } \\
\text { : } \\
0 \\
0\end{array}$ & 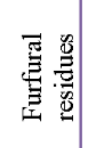 \\
\hline
\end{tabular}




\begin{tabular}{|c|c|c|c|c|c|c|c|c|}
\hline 蚫 & $\stackrel{\sqrt[n]{\varrho}}{\Theta}$ & $\begin{array}{l}\stackrel{\square}{\Xi} \\
\stackrel{\mathscr{m}}{=}\end{array}$ & $\underset{\Theta}{\stackrel{F}{\Xi}}$ & $\begin{array}{l}\bar{\infty} \\
\stackrel{=}{g}\end{array}$ & 뭉 & $\begin{array}{l}\bar{g} \\
\stackrel{9}{3}\end{array}$ & 导 & $\underset{\Xi}{\Xi}$ \\
\hline | & I & ๕̊̆ & 1 & I & : & 8 & $\underset{\infty}{\stackrel{f}{\infty}}$ & I \\
\hline | & $\infty$ & 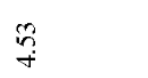 & 1 & I & तે & $\stackrel{\varrho}{\varrho}$ & 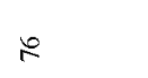 & I \\
\hline$\underset{ల}{\infty}$ & $\frac{\mathcal{F}}{m}$ & స్ & $\stackrel{8}{\circ}$ & : & | & $\overline{\bar{\lambda}}$ & $\frac{m}{m}$ & $\begin{array}{l}\stackrel{5}{+} \\
\dot{J} \\
\end{array}$ \\
\hline $\begin{array}{l}3 \\
0 \\
0 \\
0\end{array}$ & 兑 & 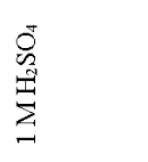 & $\begin{array}{l}3 \\
0 \\
0 \\
0\end{array}$ & 窎 & $\underset{\substack{0 \\
:}}{\sum_{n}}$ & $\sum_{m}^{:}$ & 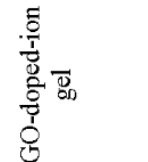 & 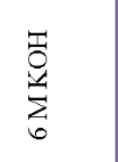 \\
\hline$n$ & $\because$ & | & - & तิ & - & तి & - & - \\
\hline$\stackrel{ }{\sim}$ & वे & ¿્ત & 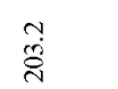 & $\stackrel{\circ}{9}$ & $\begin{array}{l}\stackrel{\infty}{0} \\
\stackrel{9}{9}\end{array}$ & $\begin{array}{c}\infty \\
\stackrel{0}{\Omega}\end{array}$ & 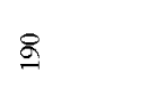 & $\stackrel{\circ}{\infty}$ \\
\hline 1 & $\stackrel{8}{\circ}$ & $\begin{array}{l}\text { \& } \\
\text { in }\end{array}$ & I & $\underset{8}{8}$ & | & \&̊ & & \\
\hline 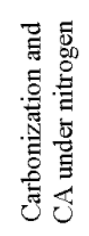 & 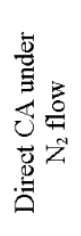 & 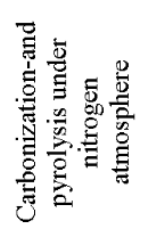 & 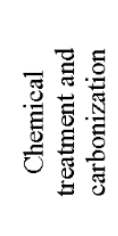 & 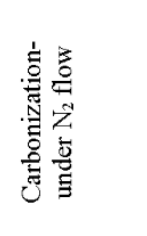 & 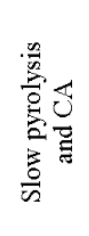 & 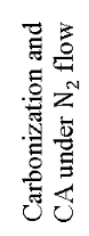 & $\begin{array}{l}\overleftrightarrow{U} \\
\vec{\Xi} \\
\tilde{E} \\
0 \\
0\end{array}$ & 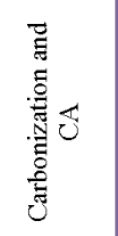 \\
\hline 密 & 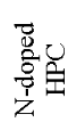 & 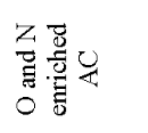 & 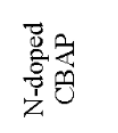 & 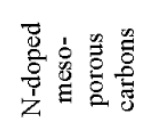 & 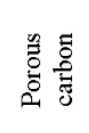 & Y & 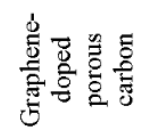 & 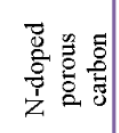 \\
\hline 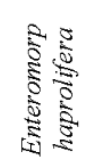 & 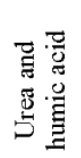 & 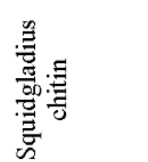 & है & 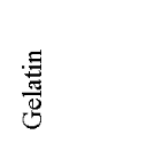 & 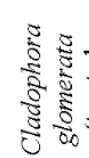 & $\begin{array}{l}\text { : } \\
\text { है }\end{array}$ & 号 & Е \\
\hline
\end{tabular}




\begin{tabular}{|c|c|c|c|c|c|c|c|c|c|}
\hline$\stackrel{g}{\stackrel{g}{\Xi}}$ & $\stackrel{\bar{g}}{\Xi}$ & 寻 & 骂 & $\underset{g}{g}$ & 焉 & 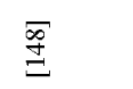 & $\Xi$ & 氶 & 홀 \\
\hline . & ๕ัે & 1 & I & $\stackrel{\circ}{\circ}$ & $\underset{\infty}{\infty}$ & 1 & 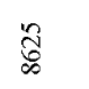 & $\underset{\mathrm{d}}{\mathrm{d}}$ & $\begin{array}{l}= \\
\overrightarrow{0} \\
\stackrel{\infty}{\infty}\end{array}$ \\
\hline 寸 & $\hat{a}$ & 1 & $\ddot{\ddot{d}}$ & $\stackrel{\sim}{F}$ & '? & I & $\stackrel{3}{\varrho}$ & $\stackrel{\infty}{\stackrel{9}{9}}$ & $\begin{array}{l}\overrightarrow{\overrightarrow{0}} \\
\stackrel{\Xi}{\Xi}\end{array}$ \\
\hline 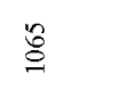 & | & I & I & 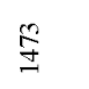 & 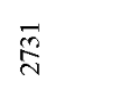 & $\stackrel{\mathscr{8}}{\stackrel{8}{J}}$ & बे & $\overline{\mathscr{D}}$ & 号 \\
\hline 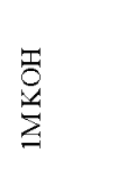 & $\begin{array}{l}3 \\
0 \\
\vdots \\
0\end{array}$ & 荾 & 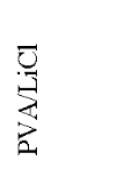 & 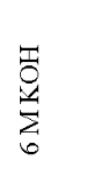 & 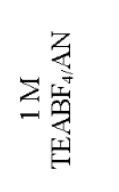 & 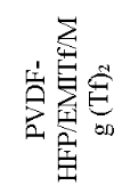 & 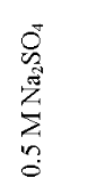 & 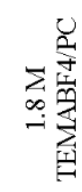 & \\
\hline | & $\because$ & $\because$ & $\because$ & $\stackrel{\sim}{0}$ & $\stackrel{n}{0}$ & I & - & - & ֻี \\
\hline$E$ & 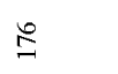 & $\cong$ & 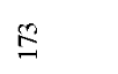 & $\stackrel{\infty}{\stackrel{\circ}{\circ}}$ & $\stackrel{\infty}{\stackrel{\circ}{\circ}}$ & $\stackrel{\infty}{\stackrel{\circ}{\circ}}$ & $\underline{\underline{6}}$ & $\underline{\underline{\sigma}}$ & $\stackrel{8}{\circ}$ \\
\hline$\S$ & $\begin{array}{l}8 \\
8 \\
\text { \&ิ }\end{array}$ & $\stackrel{8}{\circ}$ & 1 & 1 & $\mid$ & I & I & & | \\
\hline 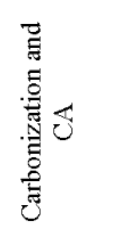 & 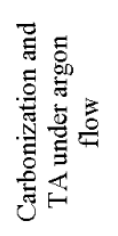 & 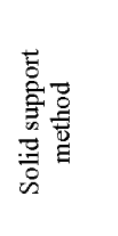 & 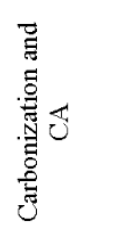 & 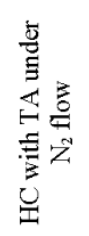 & 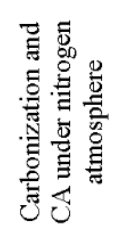 & 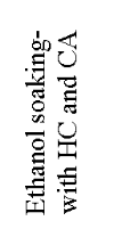 & 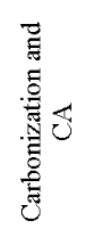 & $\begin{array}{l}\mathbb{U} \\
\widetilde{\Xi} \\
\tilde{J} \\
\tilde{u}\end{array}$ & 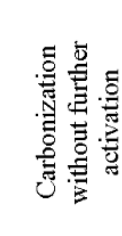 \\
\hline t & 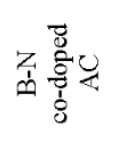 & 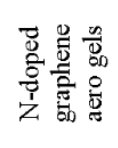 & 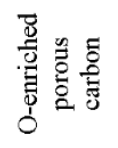 & 定 & U & Y & 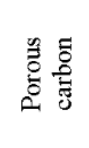 & 隹 & $\sum_{i}^{U}$ \\
\hline 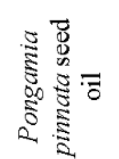 & 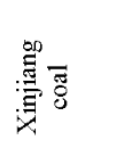 & 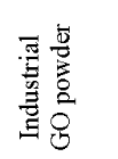 & 毁 & $\begin{array}{l}\text { 亮 } \\
\text { E⿱ } \\
8\end{array}$ & 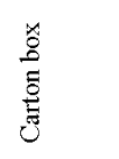 & 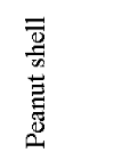 & 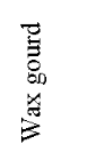 & $\begin{array}{l}\text { E } \\
\text { 总 } \\
\text { 言 } \\
\text { 产 }\end{array}$ & 递 \\
\hline
\end{tabular}




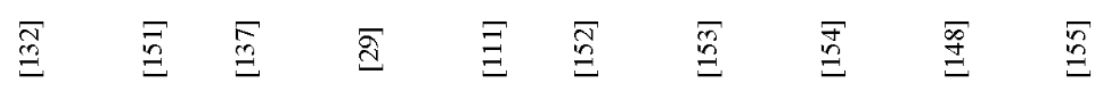

$$
\begin{aligned}
& \stackrel{n}{\Xi} \mid
\end{aligned}
$$

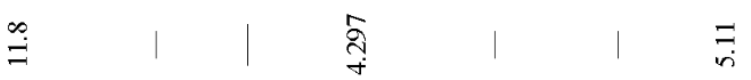

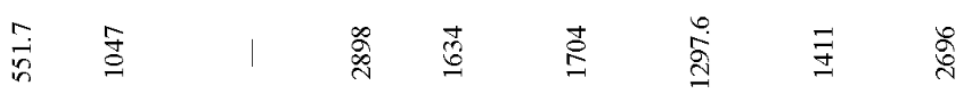

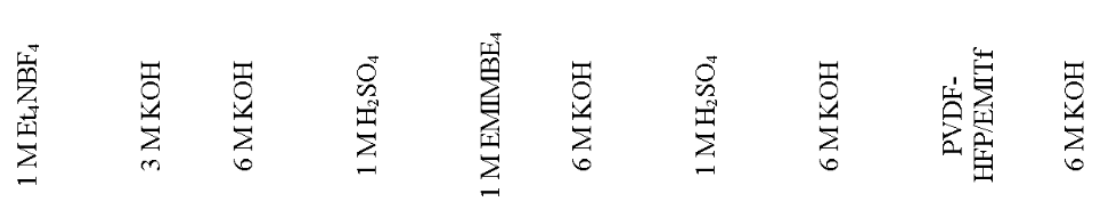

$$
\begin{aligned}
& \begin{array}{lllllllll} 
& -1 & 0 & 0 & 0 & 0 & 0 & 0 & 0
\end{array}
\end{aligned}
$$

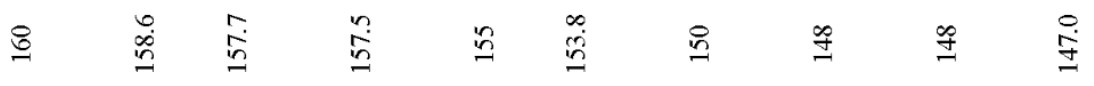

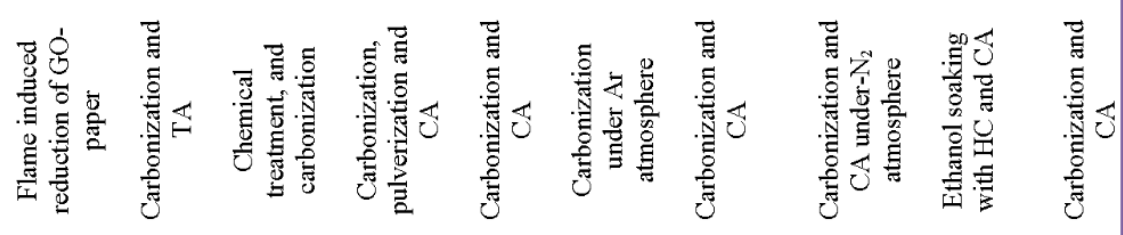

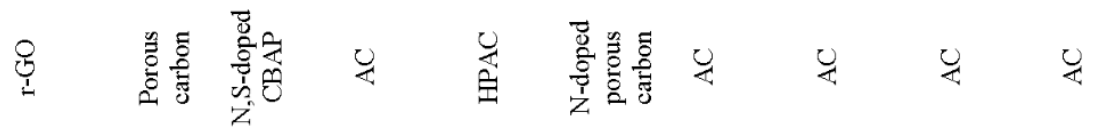

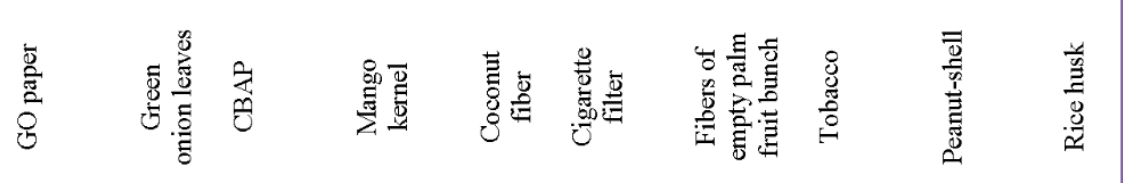




\begin{tabular}{|c|c|c|c|c|c|c|c|c|}
\hline \begin{tabular}{l}
$\square$ \\
$ٌ$ \\
\hdashline
\end{tabular} & $\stackrel{\Gamma}{\varrho}$ & $\begin{array}{l}\stackrel{\infty}{\varrho} \\
\stackrel{⿹}{=}\end{array}$ & 离 & 高 & 它 & $\underset{\widetilde{S}}{\widetilde{G}}$ & 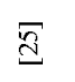 & 霫 \\
\hline$\vec{m}$ & $\mid$ & ฌั & 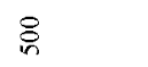 & ‡్丶 & I & | & & I \\
\hline פे & $\mid$ & $\stackrel{\vec{r}}{\circ}$ & $\stackrel{\stackrel{I}{ \pm}}{\sim}$ & से & $\begin{array}{l}\text { n़ } \\
\text { à }\end{array}$ & 1 & & 1 \\
\hline 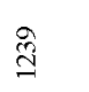 & $\begin{array}{l}\infty \\
\infty \\
\infty \\
\dot{f} \\
\dot{f}\end{array}$ & 1 & I & $\underset{\sim}{\sim}$ & 号 & $\stackrel{\infty}{\stackrel{2}{\Xi}}$ & ః્ & $\stackrel{\stackrel{一}{\oplus}}{=}$ \\
\hline $\begin{array}{l}5 \\
0 \\
\vdots \\
6\end{array}$ & 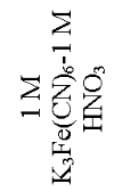 & 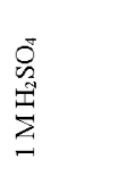 & 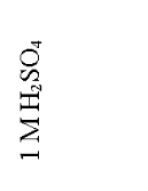 & 空 & 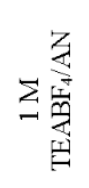 & 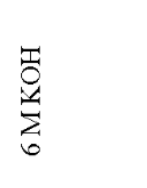 & $\begin{array}{l}0 \\
0 \\
. \\
\vdots \\
\vdots \\
=\end{array}$ & 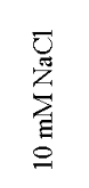 \\
\hline ؛ి & तે & - & I & - & - & $\stackrel{8}{8}$ & 1 & $\overline{0}$ \\
\hline 寻 & q & 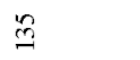 & సి & 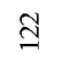 & $\cong$ & $\stackrel{\circ}{\varrho}$ & 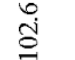 & $\approx$ \\
\hline | & $\underset{-}{8}$ & ๕. & | & $\begin{array}{l}8 \\
\cong\end{array}$ & \&̊ & $\stackrel{8}{8}$ & $\begin{array}{l}8 \\
\stackrel{\Xi}{0}\end{array}$ & | \\
\hline 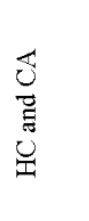 & 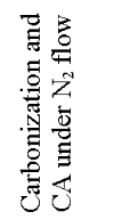 & 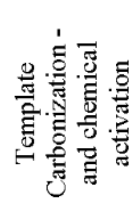 & 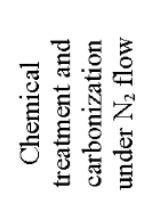 & 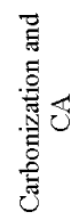 & 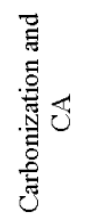 & 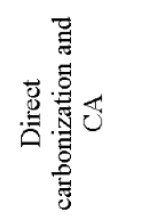 & తే & 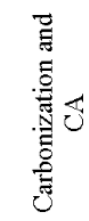 \\
\hline 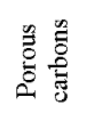 & 导 & 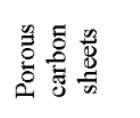 & 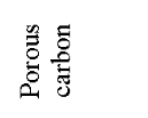 & 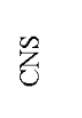 & 虽 & 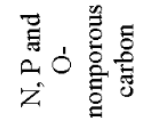 & U & U \\
\hline 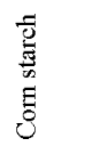 & 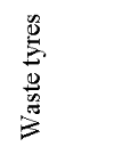 & 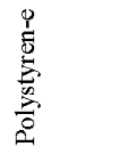 & 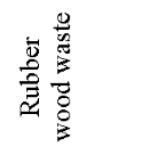 & 言 & 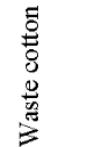 & 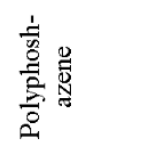 & 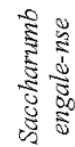 & 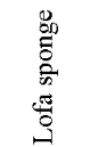 \\
\hline
\end{tabular}




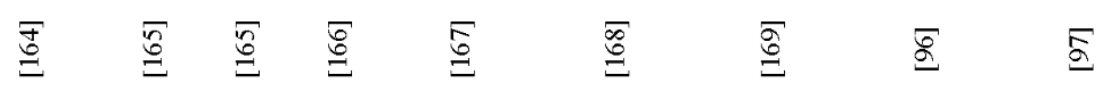

$$
\begin{aligned}
& \text { §. }
\end{aligned}
$$

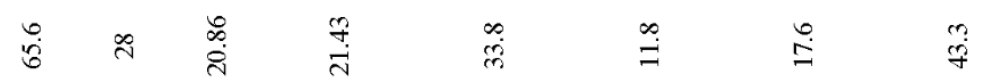

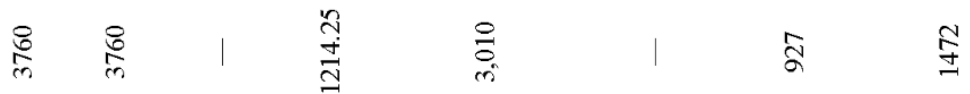

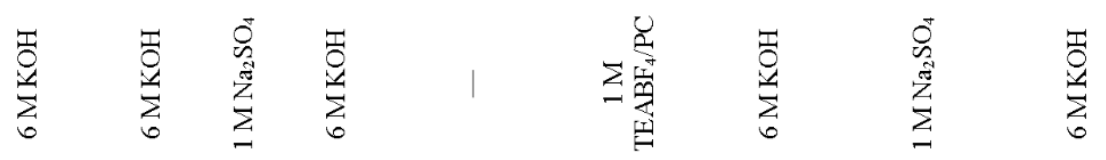

$$
\begin{aligned}
& \therefore \quad F \quad \& \quad \underset{\infty}{\infty} i
\end{aligned}
$$

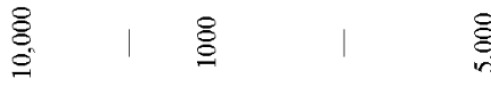

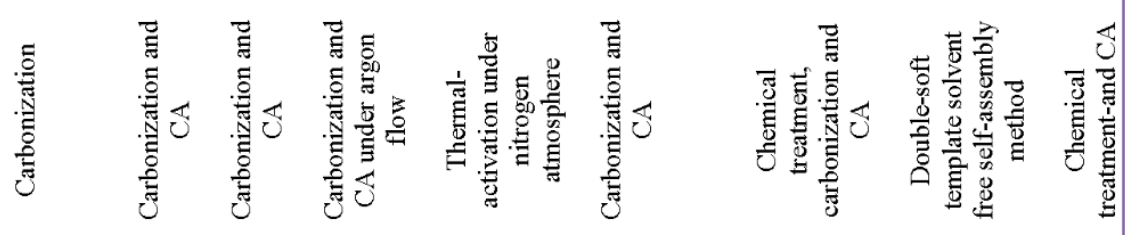

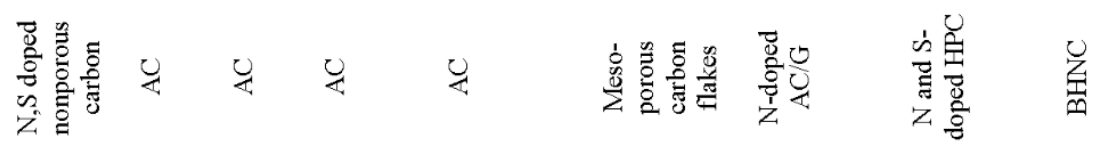

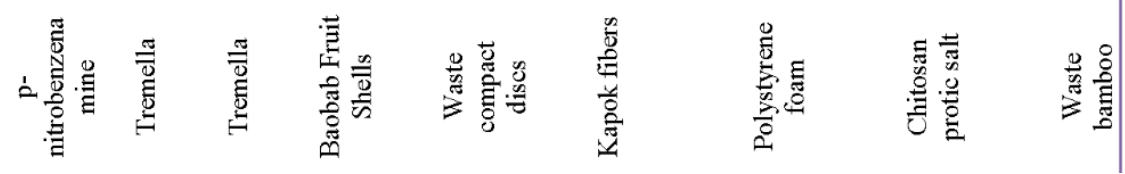




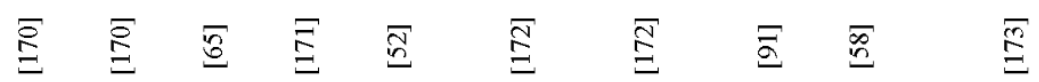

$$
\begin{aligned}
& \text { \& }
\end{aligned}
$$

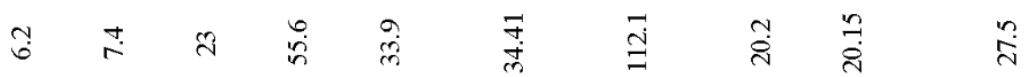

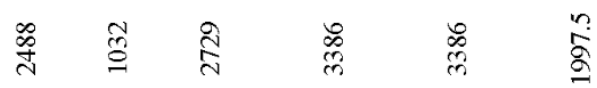

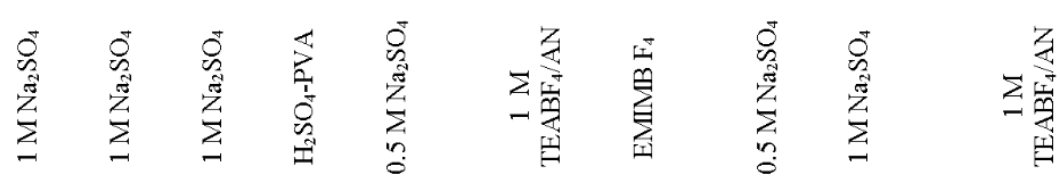

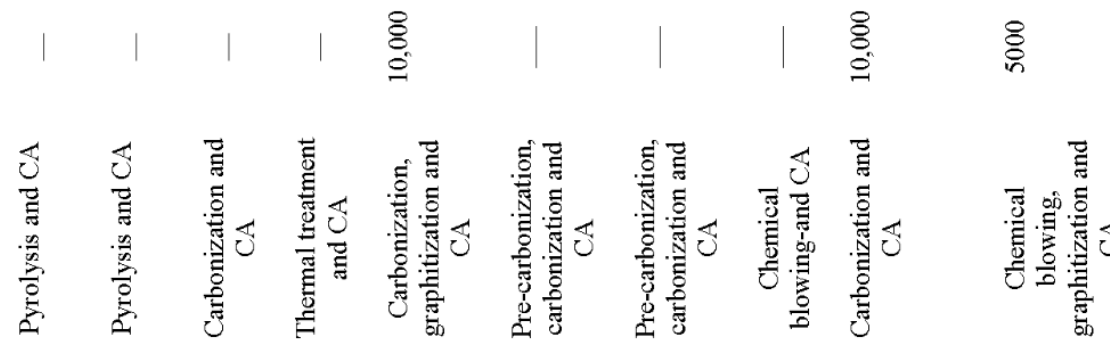

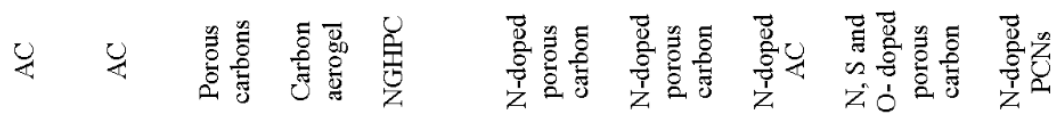

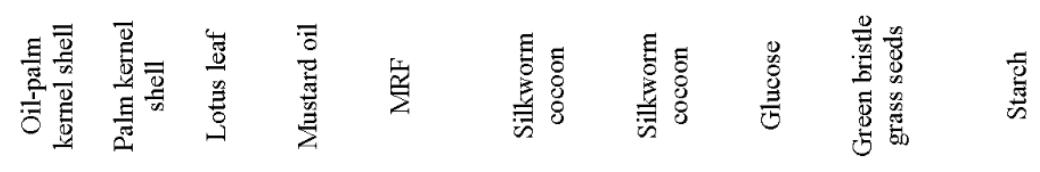




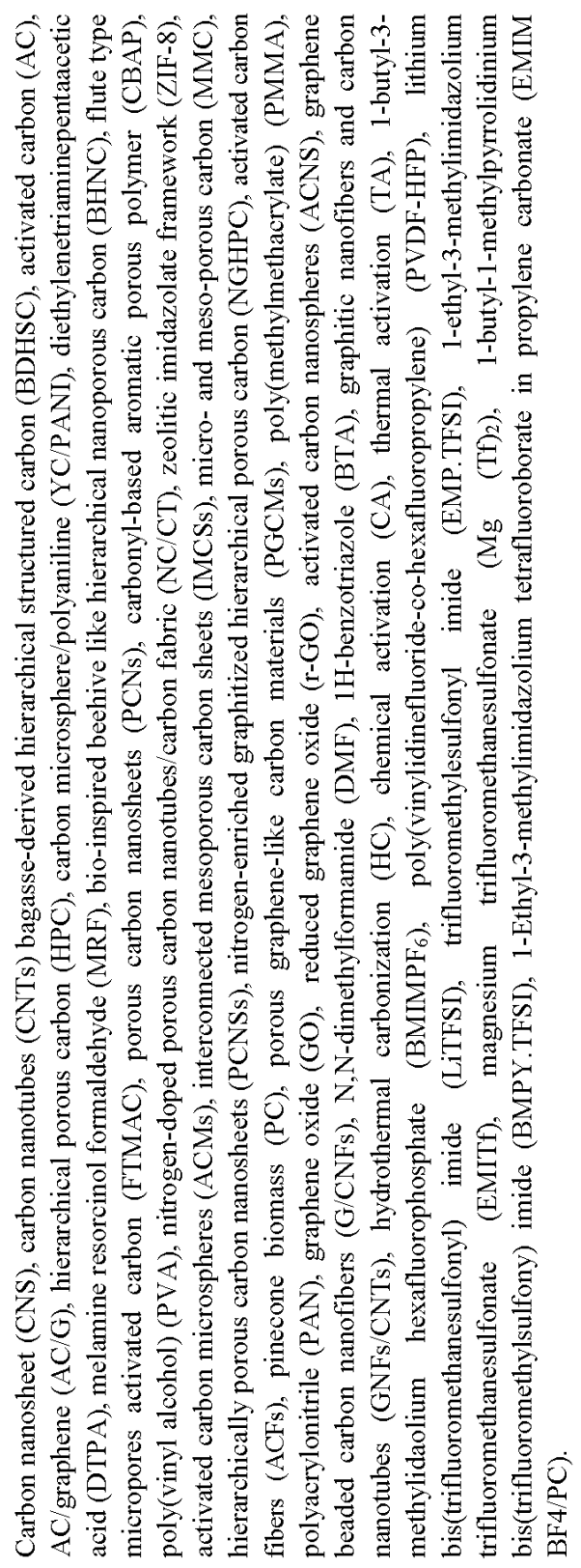




\section{Conclusion and recommendations}

It has been shown that research on energy conversion and storage, particularly using SCs, is growing progressively. A concise description of different capacitor types was given, with a focus on the non-Faradaic SCs. Review of recent carbon electrode materials with their practical performances (specific surface area, specific capacitance, energy and power densities) for non-Faradaic SCs, was made.

Development and investigation of highly active carbon materials with optimized electrolytic compatibilities and manipulative morphologies and pore structures is recommendable. In this regard, certain materials of renowned potentials are yet to be fully engaged as electrode materials for SCs and they include hemp (Cannabis sativa) and cigarette filters. A team of Canadian researchers utilized hemp fibers via hydrothermal carbonization (HC) combined with activation and found the material as efficient as graphene, in SC application [30, 160, 174]. Just afterward, "Used cigarette filters turned into supercapacitor electrodes that outperform graphene-based ones" [175], as Korean researchers utilized carbon materials prepared by a novel one-step method from cigarette filters and used it in SC application. It exhibited higher capacitance than $\mathrm{N}$-doped graphene or N-doped carbon nanotube electrodes [30, 175, 176]. These materials and the likes should be explored.

Finally, in a very recent study by Jain et al. [29], activated carbon electrode was used to achieve both EDL super-capacitance and hybrid super-capacitance, by simply adding a redox agent (p-hydroxyaniline) to the electrolyte (aqueous sulphuric acid), rather than employing the synergistic effect of different electrode materials $[4,8,177]$. This can pave the way for new designs of hybrid SCs with unique features and therefore, further investigation is recommended.

\section{Acknowledgment}

The corresponding author acknowledges full MSc. Scholarship from the Ministry of Higher Education (MOHE) at Egypt-Japan University of Science and Technology, Egypt.

\section{References}

[1] B.A. Bhanvase, V.B. Pawade, in: Nanomater. Green Energy, Elsevier, 2018, pp. 457-472.

[2] A. Yu, V. Chabot, J. Zhang, Electrochemical Supercapacitors for Energy Storage and Delivery, CRC Press Taylor \& Francis Group, 2013.

[3] G. Wang, L. Zhang, J. Zhang, Chem. Soc. Rev. 41 (2012) 797-828.

[4] Z. Abdin, Kaveh Rajab Khalilpour, in: Polygeneration with Polystorage Chem.

Energy Hubs, Academic Press, 2019, pp. 77-131.

[5] G. Xiong, A. Kundu, T.S. Fisher, Thermal Effects in Supercapacitors, 1st ed., Springer International Publishing, 2015.

[6] M. Winter, R. J. Brodd, Chem. Rev. 105 (2005) 1021-1021.

[7] Y. Wu, C. Cao, Sci. China Mater. 61 (2018) 1517.

[8] C. Zhong, Y. Deng, W. Hu, J. Qiao, L. Zhang, J. Zhang, Chem. Soc. Rev. 44 (2015)

7484-[9] Pionteck J, Wypych G (eds). Electrostatic Hazards. In: Handbook of

Antistatics. ChemTec Publishing, 2016, pp. 65-73.

[10] Von Kleist and the Leyden jar. 1905; 71. 
https://upload.wikimedia.org/wikipedia/commons/f/fa/Von_Kleist_Leyden_jar_1905.p ng (accessed 27 May 2019).

[11] S.J. Schechner, ERittenhouse 26 (2015) 1-11.

[12] Sattel S. Everything You Need to Know About Capacitors. Eagle, https://www.autodesk.com/products/eagle/blog/everything-need-know-capacitors (2016, accessed 27 May 2019).

[13] Franklin B. Experiments and Observations on Electricity: Letter IV to Peter Collinson. 1749; 38. https://www.chemteam.info/Chem-History/Franklin1749/Franklin-1749-all.pdf (accessed 27 May 2019).

[14] R. Ehiorobo, Medium Voltage Switchgear Techniques, Applicability, and Maintenance Rudiments, a MUMU (Novice) Perspective Made Simple, Dorrance Publishing, 2018. http://bookstore.dorrancepublishing.com/medium-voltage-switchgear-techniquesapplicability-and-maintenance-rudiments-a-mumu-novice-perspective-made-simple/ (accessed 27 May 2019).

[15] J. Both, IEEE Electr. Insul. Mag. 31 (2015) 24-34.

[16] Electric double layer. Merriam-Webster Dictionary, https://www.merriamwebster.com/dictionary/electric_double_layer (accessed 28 May 2019).

[17] H. Helmholtz, Ann. Phys. 165 (1853) 211-233.

[18] Samantara AK, Ratha S. Historical Background and Present Status of the Supercapacitors. In: Materials Development for Active/Passive Components of a Supercapacitor. Springer, Singapore, 2018, pp. 9-10.

[19] J.O. Bockris, M.A. V Devanathan, K. Müller, J.A.V. Butler, Proc. R. Soc. London. Ser. A. Math. Phys. Sci. 274 (1963) 55-79.

[20] Srinivasan S. Electrode/Electrolyte Interfaces: Structure and Kinetics of Charge Transfer. In: Fuel Cells: From Fundamentals to Applications. Springer-Verlag US, 2006, pp. 27-96.

[21] B.E. Conway, J. Electrochem. Soc. 138 (1991) 1539.

<a href="https://doi.org/10.1149/1.2085829" target="_blank"

rel="nofollow" $>$ Crossreff $</ a>$

[22] S.-J. Park, M.-K. Seo, Interface Sci. Technol. 18 (2011) 1-57.

[23] Woodford C. Supercapacitors, https://www.explainthatstuff.com/howsupercapacitors-work.html (2018, accessed 28 May 2019).

[24] M. Inagaki, H. Konno, O. Tanaike, J. Power Sources 195 (2010) 7880-7903.

[25] S. Rawal, B. Joshi, Y. Kumar, J. Energy Storage 20 (2018) 418-426.

[26] P. Ratajczak, M.E. Suss, F. Kaasik, F. Béguin, Energy Storage Mater. 16 (2019) 126-145.

[27] B. Viswanathan, in: Energy Sources, Elsevier, 2017, pp. 315-328.

[28] Y.-E. Miao, T. Liu, in: Electrospinning Nanofabrication Appl., William Andrew Publishing, 2019, pp. 641-669.

[29] D. Jain, J. Kanungo, S.K. Tripathi, Mater. Chem. Phys. 229 (2019) 66-77.

[30] Metalgrass LTD. Graphene Supercapacitors: Introduction and News. GrapheneInfo, https://www.graphene-info.com/graphene-supercapacitors (2019, accessed 28 May 2019).

[31] W. Zhang, S. Zhu, R. Luque, S. Han, L. Hu, G. Xu, Chem. Soc. Rev. 45 (2016) 715-752.

[32] J. Huang, Y. Liu, T. You, Anal. Methods 2 (2010) 202-211.

[33] W. Zhao, M. Fang, F. Wu, H. Wu, L. Wang, G. Chen, J. Mater. Chem. 20 (2010) 5817-5819.

[34] M.M. Titirici, R.J. White, N. Brun, V.L. Budarin, D.S. Su, F. Del Monte, J.H. 
Clark, M.J. MacLachlan, Chem. Soc. Rev. 44 (2015) 250-290.

[35] O.S. Okwundu, E.U. Aniekwe, C.E. Nwanno, Metall. Mater. Eng. 24 (2018) 145-171.

[36] D. Chen, L. Tang, J. Li, Chem. Soc. Rev. 39 (2010) 3157-3180.

[37] Y. Zhou, P. Jin, Y. Zhou, Y. Zhu, Sci. Rep. 8 (2018) 9005.

[38] Poonam, K. Sharma, A. Arora, S.K. Tripathi, J. Energy Storage 21 (2019) 801-825.

[39] X.Y. Chen, C. Chen, Z.J. Zhang, D.H. Xie, J. Mater. Chem. A 1 (2013) 10903-10911.

[40] M.D. Stoller, R.S. Ruoff, Energy Environ. Sci. 3 (2010) 1294-1301.

[41] L. Liu, Z. Niu, J. Chen, Chinese Chem. Lett. 29 (2018) 571-581.

[42] Z.S. Iro, C. Subramani, S.S. Dash, Int. J. Electrochem. Sci. 11 (2016) 10628-10643.

[43] L.L. Zhang, X.S. Zhao, Chem. Soc. Rev. 38 (2009) 2520-2531.

[44] Xu B, Hou S, Zhang F, et al. Nitrogen-doped mesoporous carbon derived from biopolymer as electrode material for supercapacitors. J Electroanal Chem 2014; 712: 146-150.

[45] N. Konikkara, L.J. Kennedy, Mater. Lett. 205 (2017) 56-61.

[46] E. Elanthamilan B. Sriram S. Rajkumar C. Dhaneshwaran N. Nagaraj J. Princy

Merlin A. Vijayan Sea-Fue Wang, Mater Res Bull, 112 (2019) 390-398.

[47] F. Gao, J. Qu, Z. Zhao, Z. Wang, J. Qiu, Electrochim. Acta, 190 (2016): 1134-1141.

[48] X. Wei, J.-S. Wei, Y. Li, H. Zou, J. Power Sources 414 (2019) 13-23.

[49] L. Lyu, H. Chai, K. dong Seong, C. Lee, J. Kang, W. Zhang, Y. Piao, Electrochim. Acta 291 (2018) 256-266.

[50] W. Singsang, M. Panapoy, B. Ksapabutr, Energy Procedia 56 (2014) 439-447.

[51] Y. Zhang, Y. Cong, J. Zhang, X. Li, Y. Li, Z. Dong, G. Yuan, J. Zhang, Z. Cui, Surf. Coatings Technol. 349 (2018) 384-391.

[52] B. Chang, W. Shi, S. Han, Y. Zhou, Y. Liu, S. Zhang, B. Yang, Chem. Eng. J., 350 (2018) 595-598

[53] M. Demir, B. Ashourirad, J.H. Mugumya, S.K. Saraswat, H.M. El-kaderi, R.B. Gupta, Int. J. Hydrogen Energy (2018) 1-10.

[54] F. Wu, J. Gao, X. Zhai, M. Xie, Y. Sun, H. Kang, Q. Tian, H. Qiu, Carbon N. Y. 147 (2019) 242-251.

[55] E. Elaiyappillai, R. Srinivasan, Y. Johnbosco, P. Devakumar, K. Murugesan, K. Kesavan, P.M. Johnson, Appl Surf Sci, 486 (2019) 527-538

[56] W. Du, X. Wang, X. Sun, J. Zhan, H. Zhang, X. Zhao, J. Electroanal. Chem. 827 (2018) 213-220.

[57] Z. Sun, M. Zheng, H. Hu, H. Dong, Y. Liang, Y. Xiao, B. Lei, Y. Liu, Chem Eng J, 336 (2018) 550-561.

[58] W. Zhou, S. Lei, S. Sun, X. Ou, Q. Fu, Y. Xu, Y. Xiao, 402 (2018) 203-212.

[59] M. Karnan, K. Subramani, P.K. Srividhya, M. Sathish, Electrochim Acta, 228(2017) 586-596.

[60] C. Shao, S. Qiu, H. Chu, Y. Zou, C. Xiang, F. Xu, L. Sun, Catal. Today 318 (2018) 150-156.

[61] Y. Liu, H. Wang, X. Kang, Y. Wang, S. Yang, S. Bian, J. Power Sources 402

(2018) 413-421.

[62] D. Tang, Y. Luo, W. Lei, Q. Xiang, W. Ren, W. Song, K. Chen, J. Sun, Appl. Surf. Sci. 462 (2018) 862-871.

[63] T. Kesavan, T. Partheeban, M. Vivekanantha, M. Kundu, G. Maduraiveeran, M. Sasidharan, Microporous Mesoporous Mater. 274 (2019) 236-244. 
[64] X. Gao, X. Li, Z. Kong, G. Xiao, Y. Zhu, Sci Bull, 63 (2018) 621-628.

[65] S. Qu, J. Wan, C. Dai, T. Jin, F. Ma, J Alloys Compd, 751 (2018) 107-116.

[66] S.E.M. Pourhosseini, O. Norouzi, H.R. Naderi, Biomass and Bioenergy 107 (2017) 287-298.

[67] J. Liu, H. Li, H. Zhang, Q. Liu, R. Li, B. Li, J. Wang, J. Solid State Chem, 257

(2017) 64-71.

[68] W. Zhang, C. Yu, L. Chang, W. Zhong, W. Yang, Electrochim. Acta, 282 (2018)

642-652.

[69] T.E. Rufford, D. Hulicova-jurcakova, Z. Zhu, G.Q. Lu, 10 (2008) 1594-1597.

[70] D. Guo, R. Xin, Y. Wang, W. Jiang, Q. Gao, G. Hu, M. Fan, Microporous

Mesoporous Mater. 279 (2019) 323-333.

[71] T.F. Zhang, Q.X. Xia, Z. Wan, J.M. Yun, Q.M. Wang, K.H. Kim, Chem. Eng. J.

360 (2019) 1310-1319.

[72] L. Chen, T. Ji, L. Mu, J. Zhu, Carbon N. Y. 111 (2017) 839-848.

[73] K. Sun, Z. Zhang, H. Peng, G. Zhao, G. Ma, Z. Lei, Mater. Chem. Phys. 218

(2018) 229-238.

[74] A. Elmouwahidi, Z. Zapata-Benabithe, F. Carrasco-Marín, C. Moreno-Castilla, Bioresour. Technol. 111 (2012) 185-190.

[75] Y. Boyjoo, Y. Cheng, H. Zhong, H. Tian, J. Pan, V.K. Pareek, S.P. Jiang, J.F. Lamonier, M. Jaroniec, J. Liu, Carbon N. Y. 116 (2017) 490-499.

[76] X.L. Su, S.H. Li, S. Jiang, Z.K. Peng, X.X. Guan, X.C. Zheng, Adv. Powder Technol. 29 (2018) 2097-2107.

[77] K. Zheng, Y. Li, M. Zhu, X. Yu, M. Zhang, L. Shi, 366 (2017) 270-277.

[78] J. Li, R. Xiao, M. Li, H. Zhang, S. Wu, C. Xia, Fuel Process. Technol. 192 (2019) 239-249.

[79] K. Wang, Y. Cao, X. Wang, M. Andrea, B. Luo, Z. Gu, J. Liu, J.D. Hoefelmeyer,

Q. Fan, 307 (2016) 462-467.

[80] J. Zhou, M. Wang, X. Li, Appl Surf Sci, 462 (2018) 444-452.

[81] J. Zhang, W. Zhang, M. Han, J. Pang, Y. Xiang, G. Cao, Microporous Mesoporous Mater. 270 (2018) 204-210.

[82] H. Chen, H. Wei, N. Fu, W. Qian, Y. Liu, H. Lin, S. Han, J Mater Sci, 53 (2018) 2669-2684.

[83] H. Zhao, B. Xing, C. Zhang, G. Huang, Q. Liu, G. Yi, J. Jia, M. Ma, Z. Chen, C.

Zhang, J Alloys Compd, 766 (2018) 705-715.

[84] T. Wu, L. Sun, F. Xu, D. Cai, J Mater Sci Technol, 34 (2018) 2384-2391.

[85] D. Wang, Z. Xu, Y. Lian, C. Ban, H. Zhang, J. Colloid Interface Sci. 542 (2019) 400-409.

[86] M. Fu, W. Chen, J. Ding, X. Zhu, Q. Liu, W. Chen, J. Ding, X. Zhu, Q. Liu, J

Alloys Compd, 782 (2018) 952-960.

[87] H. Feng, H. Hu, H. Dong, Y. Xiao, Y. Cai, B. Lei, J. Power Sources 302 (2016) 164-173.

[88] Z. Lin, X. Xiang, S. Peng, X. Jiang, L. Hou, J. Electroanal. Chem. 823 (2018) 563-572.

[89] L. Yao, G. Yang, P. Han, Z. Tang, J. Yang, J. Power Sources 315 (2016) 209-217.

[90] Z. Xu, J. Chen, X. Zhang, Q. Song, J. Wu, L. Ding, C. Zhang, H. Zhu, H. Cui,

Microporous Mesoporous Mater. 276 (2019) 280-291.

[91] Y. Wang, H. Xuan, G. Lin, F. Wang, Z. Chen, X. Dong, J. Power Sources 319 (2016) 262-270.

[92] Z. Xu, Y. Li, D. Li, D. Wang, J. Zhao, Z. Wang, M.N. Banis, Y. Hu, H. Zhang, Appl. Surf. Sci. 444 (2018) 661-671. 
[93] X. Li, J. Zhao, Z. Cai, F. Ge, Mater. Res. Bull. 107 (2018) 355-360.

[94] S. Du, L. Wang, X. Fu, M. Chen, C. Wang, Bioresour. Technol. 139 (2013) 406-409.

[95] L. Zhu, Q. Gao, Y. Tan, W. Tian, J. Xu, K. Yang, C. Yang, Microporous

Mesoporous Mater. 210 (2015) 1-9.

[96] L. Sun, Y. Zhou, L. Li, H. Zhou, X. Liu, Q. Zhang, B. Gao, 468 (2019) 382-390.

[97] Tian, Weiqian, Qiuming Gao, Yanli Tan, Kai Yang, Lihua Zhu, Chunxiao Yang,

and Hang Zhang, Journal of Materials Chemistry A, 3 (2015) 5656-5664.

[98] B. Wang, Y. Wang, Y. Peng, X. Wang, J. Wang, J. Zhao, J. Power Sources 390

(2018) 186-196.

[99] Y. Li, G. Wang, T. Wei, Z. Fan, P. Yan, Nano Energy, 19 (2015) 165-175.

[100] M. Liu, F. Wei, X. Yang, S. Dong, Y. Li, X. He, New Carbon Mater. 33 (2018) 316-323.

[101] J. Li, S. Ma, L. Cheng, Q. Wu, Mater. Lett. 139 (2015) 429-432.

[102] Y. Zhou, J. Ren, Y. Yang, Q. Zheng, J. Liao, F. Xie, W. Jie, D. Lin, J. Solid State

Chem. 268 (2018) 149-158.

[103] Zhang, Wenli, Mingzhu Zhao, Ruiyi Liu, Xiaofeng Wang, and Haibo Lin: Colloids and Surfaces A: Physicochemical and Engineering Aspects, 484 (2015): 518-527.

[104] Y. Zhao, M. Lu, P. Tao, Y. Zhang, X. Gong, Z. Yang, G. Zhang, H. Li, J. Power Sources 307 (2016) 391-400.

[105] F. Chen, Q. Lin, J. Mater. Sci. Technol. (2018).

[106] Y. Cai, Y. Luo, H. Dong, X. Zhao, Y. Xiao, Y. Liang, H. Hu, Y. Liu, M. Zheng, J. Power Sources 353 (2017) 260-269.

[107] F. Barzegar, A. Bello, J.K. Dangbegnon, N. Manyala, X. Xia, Energy Procedia 105 (2017) 4098-4103.

[108] Z.Q. Hao, J.P. Cao, Y. Wu, X.Y. Zhao, L. Zhou, X. Fan, Y.P. Zhao, X.Y. Wei, Fuel Process. Technol. 162 (2017) 45-54.

[109] J. Lei, J. Zhou, J. Li, J. Wen, L. Su, T. Duan, W. Zhu, Electrochim. Acta 285 (2018) 292-300.

[110] S. Zhang, X. Shi, R. Wróbel, X. Chen, E. Mijowska, Electrochim. Acta 294

(2019) 183-191.

[111] L. Yin, Y. Chen, D. Li, X. Zhao, B. Hou, B. Cao, 111 (2016) 44-50.

[112] Z. Wang, S. Yun, X. Wang, C. Wang, Y. Si, Y. Zhang, H. Xu, Ceram Int, 45

(2018) 4208-4218.

[113] Z. Zhou, X. Wu, J. Power Sources 222 (2013) 410-416.

[114] Q. Xiong, Q. Bai, C. Li, D. Li, X. Miao, Y. Shen, J Taiwan Inst Chem Eng 95 (2019) 315-323.

[115] M.-M. Wei, W.-P. Li, J. Weng, Y.-Q. Liu, S.-R. Li, Y.-Y. Ye, M. Wang, D.

Wang, J Saudi Chem Soc, In Press (2019).

[116] Y. Yang, L. Liu, Y. Tang, Y. Zhang, D. Jia, L. Kong, Electrochim. Acta 191 (2016) 846-853.

[117] D. Yu, Y. Ma, M. Chen, X. Dong, J Colloid Interface Sci, 537 (2018) 569-578.

[118] M.Y. Bhat, N. Yadav, S.A. Hashmi, Electrochim. Acta 304 (2019) 94-108.

[119] X. Xu, J. Gao, Q. Tian, X. Zhai, Y. Liu, Appl. Surf. Sci. 411 (2017) 170-176.

[120] X. Tian, H. Ma, Z. Li, S. Yan, L. Ma, F. Yu, G. Wang, X. Guo, Y. Ma, C. Wong, J. Power Sources 359 (2017) 88-96.

[121] L. Wei, K. Tian, Y. Jin, X. Zhang, X. Guo, Microporous Mesoporous Mater. 227 (2016) 210-218. 
[122] X.T. Wang, H. Ma, X.J. He, J.X. Wang, J.F. Han, Y. Wang, Xinxing Tan Cailiao/New Carbon Mater. 32 (2017) 213-220.

[123] Z. Wang, Y. Tan, Y. Yang, X. Zhao, Y. Liu, L. Niu, B. Tichnell, L. Kong, L. Kang, Z. Liu, F. Ran, J. Power Sources 378 (2018) 499-510.

[124] Y. Li, X. Wang, M. Cao, J. CO2 Util. 27 (2018) 204-216.

[125] A.K. Mondal, K. Kretschmer, Y. Zhao, H. Liu, H. Fan, G. Wang, Microporous Mesoporous Mater. 246 (2017) 72-80.

[126] M. Nazarian-samani, S. Haghighat-shishavan, M. Nazarian-samani, M. Kim, B. Cho, S. Oh, S.F. Kashani-bozorg, K. Kim, J. Power Sources 372 (2017) 286-296.

[127] L. Wang, Q. Zhu, J. Zhao, Y. Guan, J. Liu, Z. An, B. Xu, Microporous Mesoporous Mater. 279 (2019) 439-445.

[128] P. Zhang, F. Sun, Z. Shen, D. Cao, Journal of Materials Chemistry A, 32 (2014) 12873-12880.

[129] F. Zeng, Z. Li, X. Li, J. Wang, Z. Kong, Y. Sun, Z. Liu, Appl. Surf. Sci. 467-468 (2019) 229-235

[130] J. Zhang, W. Zhang, H. Zhang, J. Pang, G. Cao, J. Alloys Compd. 712 (2017) 76-81.

[131] J. Hou, K. Jiang, M. Tahir, X. Wu, F. Idrees, M. Shen, C. Cao, J. Power Sources 371 (2017) 148-155.

[132] D. Sun, X. Yan, J. Lang, Q. Xue, J. Power Sources 222 (2013) 52-58.

[133] Y. Yin, Y. Gao, Y. Zhang, A. Li, G. Ji, Microporous Mesoporous Mater. 278 (2019) 195-205.

[134] Y. Gao, W. Zhang, Q. Yue, B. Gao, Y. Sun, J. Kong, P. Zhao, J. Power Sources 270 (2014) 403-410.

[135] Y. Zhu, M. Chen, Y. zhang, W. Zhao, C. Wang, Carbon N. Y. 140 (2018) 404-412.

[136] C.J. Raj, M. Rajesh, R. Manikandan, K. Hyun, J.R. Anusha, J. Hwan, D. Kim, S. Yeup, B. Chul, J. Power Sources 386 (2018) 66-76.

[137] M. Kim, P. Puthiaraj, J. So, H. Seong, J. Ryu, W. Ahn, Synth. Met. 243 (2018) 115-120.

[138] [138] B. Xu, S. Hou, F. Zhang, G. Cao, M. Chu, Y. Yang, J. Electroanal. Chem. 712 (2014) 146-150.

[139] H. Guo, Mater Res Bull, 102 (2018) 391-398.

[140] X. Yang, L. Zhang, F. Zhang, T. Zhang, Y. Huang, Y. Chen, Carbon N. Y. 72 (2014) 381-386.

[141] E. Lei, W. Li, C. Ma, Z. Xu, S. Liu, Appl. Surf. Sci. 457 (2018) 477-486.

[142] K. Chaitra, R. Narendra, K. Venkatesh, N. Nagaraju, N. Kathyayini, J. Power Sources 356 (2017) 212-222.

[143] Q. Lu, Y.Y. Xu, S.J. Mu, W.C. Li, Xinxing Tan Cailiao/New Carbon Mater. 32 (2017) 442-450.

[144] J. Du, L. Liu, Y. Yu, L. Zhang, Y. Zhang, A. Chen, Mater. Chem. Phys. 223 (2019) 145-151.

[145] W. Chen, X. Wang, M. Feizbakhshan, C. Liu, S. Hong, P. Yang, X. Zhou, J. Colloid Interface Sci. 540 (2019) 524-534.

[146] W. Cao, F. Yang, Mater. Today Energy 9 (2018) 406-415.

[147] D. Wang, G. Fang, T. Xue, J. Ma, G. Geng, J. Power Sources 307 (2016) 401-409.

[148] N. Yadav, M.K. Singh, N. Yadav, S.A. Hashmi, J. Power Sources 402 (2018) 133-146.

[149] W. Sun, S.M. Lipka, C. Swartz, D. Williams, F. Yang, Carbon, 103 (2016) 181-192.

[150] X. Tian, S. Zhu, J. Peng, Y. Zuo, G. Wang, X. Guo, N. Zhao, Y. Ma, L. Ma, Electrochim. Acta 241 (2017) 170-178. 
[151] J. Yu, L. Gao, X. Li, C. Wu, L. Gao, C. Li, New Carbon Mater. 31 (2016) 475-484. [152] M. Lee, G. Kim, H.D. Song, S. Park, Nanotechnology, 25 (2014) 345601. [153] R. Farma, M. Deraman, A. Awitdrus, I.A. Talib, E. Taer, N.H. Basri, Bioresour. Technol. 132 (2013) 254-261.

[154] H. Chen, Y. Guo, F. Wang, G. Wang, P. Qi, X. Guo, B. Dai, New Carbon Mater. 32 (2017) 592-599.

[155] E. Yi, L. Teo, L. Muniandy, E. Ng, F. Adam, A.R. Mohamed, R. Jose, K.F. Chong, Electrochim Acta, 192 (2016) 110-119.

[156] L. Pang, B. Zou, Y. Zou, X. Han, L. Cao, W. Wang, Y. Guo, Colloids Surfaces A Physicochem. Eng. Asp. 504 (2016) 26-33.

[157] A. Bello, D.Y. Momodu, M.J. Madito, K. Makgopa, K.M. Rambau, J.K. Dangbegnon, N.M. Musyoka, N. Manyala, 209 (2018) 262-270.

[158] Y. Wen, X. Wen, K. Wenelska, X. Chen, E. Mijowska, Diam. Relat. Mater. 95 (2019) 5-13.

[159] U. Thubsuang, S. Laebang, N. Manmuanpom, S. Wongkasemjit, J Mater Sci, 52 (2017) 6837-6855.

[160] H. Wang, Z. Xu, A. Kohandehghan, Z. Li, K. Cui, X. Tan, T. James Stephenson, C. K. King'ondu, C. M. B. Holt, B. C. Olsen, J. Kwon Tak, D. Harfield, A. O. Anyia, D. Mitlin, ACS Nano 7 (2013) 5131-5141.

[161] M. Vijayakumar, R. Santhosh, J. Adduru, T.N. Rao, M. Karthik, Carbon, 140 (2018) 465-476.

[162] W. Liu, S. Zhang, S.U. Dar, Y. Zhao, R. Akram, X. Zhang, S. Jin, Z. Wu, D. Wu, Carbon, 129 (2018) 420-427.

[163] C. Feng, Y. Chen, C. Yu, C. Hou, Chemosphere, 208 (2018) 285-293.

[164] H.T. Yi, Y.Q. Zhu, X.Y. Chen, Z.J. Zhang, J Alloys Compd, 649 (2015) 851-858.

[165] N. Guo, M. Li, X. Sun, F. Wang, R. Yang, Mater. Chem. Phys. 201 (2017) 399-407.

[166] A.A. Mohammed, C. Chen, Z. Zhu, J. Colloid Interface Sci. 538 (2019) 308-319.

[167] R. Farzana, R. Rajarao, B.R. Bhat, V. Sahajwalla, J Ind Eng Chem, 65 (2018) 387-396.

[168] Z. Zou, T. Liu, C. Jiang, Mater. Chem. Phys. 223 (2019) 16-23.

[169] Q. Xie, G. Chen, R. Bao, Y. Zhang, S. Wu, Microporous Mesoporous Mater. 239 (2017) 130-137.

[170] Izan Izwan Misnon, Nurul, K. Mohd, J. Rajan, Waste and Biomass Valorization (2019) 1731-1740.

[171] V. Sahu, R.B. Marichi, G. Singh, R.K. Sharma, Electrochim. Acta 240 (2017) 146-154.

[172] J. Sun, J. Niu, M. Liu, J. Ji, M. Dou, F. Wang, Appl. Surf. Sci. 427 (2018) 807-813.

[173] B. Chang, H. Yin, X. Zhang, S. Zhang, B. Yang, Chem Eng J, 312 (2017) 191-203.

[174] Morgan J. Hemp fibres 'better than graphene'. Science reporter, BBC News, San Francisco, https://www.bbc.com/news/science-environment-28770876 (2014), accessed 29 May 2019).

[175] Metalgrass LTD. Used cigarette filters turned into supercapacitor electrodes that outperform graphene-based ones. Graphene-info, https://www.graphene-info.com/usedcigarette-filters-turned-supercapacitor-electrodes-outperform-graphene-based-ones (2019, accessed 29 May 2019).

[176] M. Lee, G.-P. Kim, H. Don Song, S. Park, J. Yi, Nanotechnology 25 (2014) 345601.

[177] A. Vlad, N. Singh, J. Rolland, S. Melinte, P.M. Ajayan, J.-F. Gohy, Sci. Rep. 4 (2014) 4315.

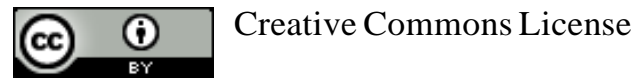

This work is licensed under a Creative Commons Attribution 4.0 International License. 